\title{
Three-dimensional instabilities in compressible flow over open cavities
}

\author{
GUILLAUME A. BRÈS AND TIM COLONIUS \\ Department of Mechanical Engineering, California Institute of Technology, \\ 1200 E. California Blvd., Pasadena, CA 91125, USA
}

(Received 26 March 2007 and in revised form 30 October 2007)

Direct numerical simulations are performed to investigate the three-dimensional stability of compressible flow over open cavities. A linear stability analysis is conducted to search for three-dimensional global instabilities of the two-dimensional mean flow for cavities that are homogeneous in the spanwise direction. The presence of such instabilities is reported for a range of flow conditions and cavity aspect ratios. For cavities of aspect ratio (length to depth) of 2 and 4, the three-dimensional mode has a spanwise wavelength of approximately one cavity depth and oscillates with a frequency about one order of magnitude lower than two-dimensional Rossiter (flow/acoustics) instabilities. A steady mode of smaller spanwise wavelength is also identified for square cavities. The linear results indicate that the instability is hydrodynamic (rather than acoustic) in nature and arises from a generic centrifugal instability mechanism associated with the mean recirculating vortical flow in the downstream part of the cavity. These three-dimensional instabilities are related to centrifugal instabilities previously reported in flows over backward-facing steps, lid-driven cavity flows and Couette flows. Results from three-dimensional simulations of the nonlinear compressible Navier-Stokes equations are also reported. The formation of oscillating (and, in some cases, steady) spanwise structures is observed inside the cavity. The spanwise wavelength and oscillation frequency of these structures agree with the linear analysis predictions. When present, the shear-layer (Rossiter) oscillations experience a low-frequency modulation that arises from nonlinear interactions with the threedimensional mode. The results are consistent with observations of low-frequency modulations and spanwise structures in previous experimental and numerical studies on open cavity flows.

\section{Introduction}

From the canonical rectangular cut-out to more complicated shapes with internal structures, resonant cavity instabilities are endemic to a number of aircraft components, including weapon bays, landing gear wells and instrumentation cavities. Selfsustained oscillations and intense acoustic loading inside the cavity can lead to structural damage, optical distortion and store separation problems. In compressible flow, cavity oscillations are typically described as a flow-acoustic resonance mechanism: small instabilities in the shear layer interact with the downstream corner of the cavity and generate acoustic waves, which propagate upstream and create new disturbances in the shear layer. The feedback process leads to reinforcement, and ultimately saturation, at one or more resonant frequencies. This type of instability is referred to as shear-layer (or Rossiter) mode, from the early work of Rossiter 
(1964) who developed the now classic semi-empirical formula to predict the resonant frequencies:

$$
S t=\frac{f L}{U}=\frac{n-\alpha}{M+1 / \kappa},
$$

where $n=1,2, \ldots$ leads to the Strouhal number $S t$ of mode I, II, etc. The empirical constants $\kappa$ and $\alpha$ correspond to the average convection speed of the vortical disturbances in the shear layer, and a phase delay, respectively.

Over the past few decades, two-dimensional cavity flows have received significant attention (see for instance review articles by Rockwell \& Naudascher 1978; Colonius 2001; Rowley \& Williams 2006). Aside from numerical benchmarking, the main motivations for studying cavity flow are noise reduction and flow control. Fundamental research has been conducted recently to examine how active (openand closed-loop) flow control can be use to replace traditional passive devices such as spoilers, ramps and rakes (e.g. Cattafesta et al. 1999; Alvarez, Kerschen \& Tumin 2004; Rowley et al. 2006; Rowley \& Williams 2006). Model-based closed-loop control, in particular, promises efficient (low energy input) tone suppression, while passive devices may be more effective for broadband noise reductions. However, past efforts in this regard have typically ignored non-parallel and three-dimensional effects that may, on one hand, reduce the effectiveness of model-based control, or on the other hand disregard important three-dimensional mechanisms that could be exploited in passive ways to reduce broadband noise.

Recently, some aspects of the three-dimensional cavity flow have been investigated using large-eddy simulation (LES) (Rizzetta \& Visbal 2003; Larchevêque et al. 2004; Chang, Constantinescu \& Park 2006) and proper orthogonal decomposition (POD) (Podvin et al. 2006). These studies have been focused on the frequencies of oscillation and coherence of the (two-dimensional) Rossiter modes, and the extent to which there is agreement with experimental measurements of mean flow and spectra. Some observations regarding the three-dimensionality of the large-scale turbulent structures are also reported. Likewise, experiments by Maull \& East (1963), Rockwell \& Knisely (1980), Neary \& Stephanoff (1987), Forestier, Jacquin \& Geffroy (2003) and Faure et al. (2007) all reported three-dimensional structures in the flow. The origin of these three-dimensional features has not yet been understood.

Therefore, the focus of the present work is to characterize the basic instabilities of three-dimensional open cavity flows. Because the basic (steady or time-averaged) cavity flow is complex and non-parallel, our stability analysis is focused on extracting global instabilities from direct numerical simulations (DNS) of the full and linearized compressible Navier-Stokes equations. We consider two- and three-dimensional instabilities to basic cavity flows that are homogeneous in the spanwise direction. The numerical methods used are presented in $\S 2$.

The remainder of the paper is organized as follows. First, the onset of twodimensional cavity instability is characterized as a function of Reynolds number, Mach number, cavity aspect ratio, and incident shear-layer thickness. The two-dimensional modes are consistent, both in terms of oscillation frequency and eigenfunction structure, with the typical Rossiter flow/acoustic resonant modes that have been observed in many cavity experiments and flight tests. For basic cavity flows that are two-dimensionally stable, we then search for three-dimensional instabilities of the steady base flow, and identify, for the first time, the presence of such instabilities (§3). For cavity length-to-depth ratios of 1,2 and 4 considered here, the instability appears to arise from a generic centrifugal instability mechanism associated with 
the internal recirculation vortical flow that occupies the downstream part of the cavity $(\S 4)$. Next, a few selected three-dimensional numerical simulations of the full compressible Navier-Stokes equations are performed. The results (§5) exhibit threedimensional structures in good agreement with the linear analysis predictions. Finally, the connections between the three-dimensional modes we report here and the flow structures observed in previous numerical studies and experiments are discussed in $\S 6$.

\section{Numerical methods and stability analysis}

\subsection{Direct numerical simulations}

Following previous work of Rowley, Colonius \& Basu (2002) on cavity flows, we developed a DNS code to solve the full compressible Navier-Stokes (NS) equations and study the flow over three-dimensional open cavities. The equations are solved directly, meaning that no turbulence model is used and all the scales of the flow are resolved. A linearized version of the equations was also implemented, and the existing DNS code can solve linear or nonlinear NS equations for both two- and three-dimensional flows.

The flow equations are solved on a structured mesh, using a sixth-order compact finite-difference scheme for spatial discretization in the $x$ - and $y$-direction (Lele 1992) and a fourth-order Runge-Kutta algorithm for time-marching. The cavity is assumed homogeneous (periodic) in the spanwise direction ( $z$-direction) and the derivatives are computed using fast Fourier transform (FFT) with subroutines provided by the FFTW library (e.g. Frigo \& Johnson 2007). Each spanwise wavenumber is discretized on a stretched Cartesian grid, with clustering of points near the walls and the shear layer in the cavity. The boundary conditions are non-reflective for the inflow and outflow, no slip and constant temperature $\left(T=T_{\infty}\right)$ at the walls (Thompson 1990; Poinsot \& Lele 1992). In addition, a buffer zone is implemented at the inflow, outflow and normal computational boundaries to reduce acoustic reflections (Colonius, Lele \& Moin 1993; Freund 1997). Unless stated otherwise, the simulations are initiated with a Blasius flat-plate boundary layer spanning the cavity and zero flow within the cavity. The code can handle any type of block geometry and is fully parallelized using message-passing interface (MPI).

\subsection{Simulation parameters and flow conditions}

The cavity configuration and flow conditions are controlled by the following parameters: the cavity aspect ratio $L / D$ and spanwise extent $\Lambda / D$, the ratio of the cavity length to the initial boundary layer momentum thickness at the leading edge of the cavity $L / \theta_{0}$, the Reynolds number $\operatorname{Re}_{\theta}=U \theta_{0} / v$ and the free-stream Mach number $M=U / a_{\infty}$ (see figure 1). Typical grid sizes ranged from a few hundred thousand to several million grid points for two- and three-dimensional simulations, respectively. In each case, the computational domain extends several cavity depths upstream, downstream and above the cavity.

Our study focuses on cavity flows at subsonic speed $(0.1 \leqslant M \leqslant 0.8)$ and low Reynolds number $\left(35 \leqslant R e_{\theta} \leqslant 400\right)$, with laminar upstream boundary layers. For the shallow cavities considered here $(L / D=1,2$ and 4$)$, the main two-dimensional instability mechanism is the Rossiter aeroacoustic coupling, rather than the acoustic modes of the cavity, even at low subsonic speed. The onset of a Rossiter mode as a function of the parameters is summarized qualitatively as follows (Rowley et al. 2002): there is a critical value of $M, R e_{\theta}$, and $L / \theta_{0}$ beyond which oscillations occur. For low Reynolds number and Mach number, the flow is subcritical and ultimately reaches 


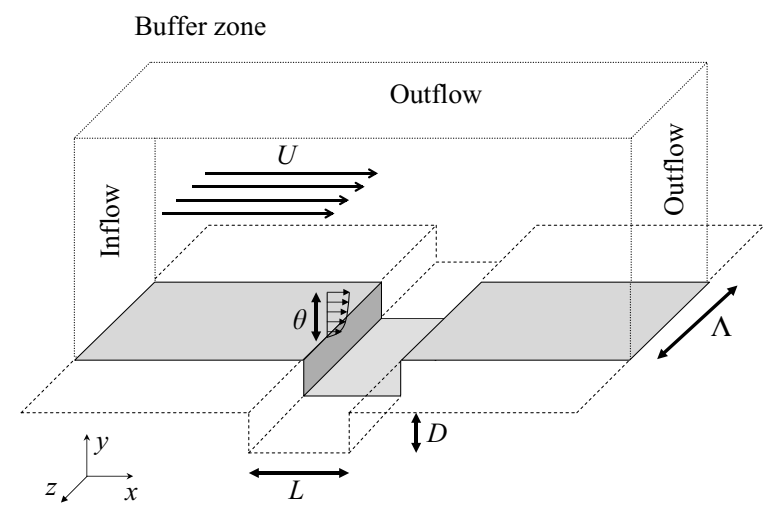

FIGURE 1. Schematic diagram of the computational domain.

a steady state. As these parameters, or $L / \theta_{0}$, are increased, the two-dimensional flow becomes supercritical and oscillates in shear-layer (Rossiter) mode.

\subsection{Linear stability theory}

To take into account non-parallel effects and possible coupling of the shear layer with the acoustic scattering and recirculating flow inside the cavity, the so-called bi-global linear stability theory has been used to study the stability of cavity flow (Theofilis \& Colonius 2003; Theofilis, Duck \& Owen 2004). In this approach, the transient solution of the equations of motion $\boldsymbol{q}=[\rho u, \rho v, \rho w, \rho, e]^{T}$ is decomposed into

$$
\boldsymbol{q}(x, y, z, t)=\overline{\boldsymbol{q}}(x, y)+\boldsymbol{q}^{\prime}(x, y, z, t),
$$

where $\overline{\boldsymbol{q}}(x, y)$ is the unknown steady two-dimensional basic flow and $\boldsymbol{q}^{\prime}(x, y, z, t)$ an unsteady three-dimensional perturbation with $\left\|\boldsymbol{q}^{\prime}\right\| \ll\|\overline{\boldsymbol{q}}\|$. As the domain is homogeneous in the spanwise direction, a general perturbation can be decomposed into Fourier modes with spanwise wavenumbers $\beta$. At linear order, modes with different wavenumber are decoupled and the following eigenmode Ansatz can be introduced:

$$
\boldsymbol{q}^{\prime}(x, y, z, t)=\sum_{n} \hat{\boldsymbol{q}}_{n}(x, y) \exp \left[\mathrm{i}\left(\beta z-\Omega_{n} t\right)\right]+\text { c.c. },
$$

where the parameter $\beta$ is taken to be a real and prescribed spanwise wavenumber, related to a spanwise wavelength in the cavity by $\lambda=2 \pi / \beta, \hat{\boldsymbol{q}}_{n}$ and $\Omega_{n}=\omega_{n}+\mathrm{i} \sigma_{n}$ are the unknown complex eigenmodes and corresponding complex eigenvalues, both dependent on $\beta$. Complex conjugation is required in (2.2) since $\boldsymbol{q}^{\prime}$ is real. The frequency and the growth/damping rate of the mode are given by $\omega_{n}$ and $\sigma_{n}$, respectively. The long-time behaviour of the linear solution will be dictated by the mode with eigenvalue $\Omega=\omega+\mathrm{i} \sigma$ of largest imaginary part. The flow is said to be subcritical (stable) if $\sigma$ is strictly negative, neutrally stable if $\sigma=0$, and supercritical (unstable) if $\sigma>0$.

The determination of the least damped (or most unstable) modes for a given wavelength $\beta$ amounts to finding the eigenvalue $\Omega$ and corresponding eigenvector, by integrating the governing equations directly in the time domain. More details on the linear stability analysis and the residual algorithm used to determine the dominant eigenmode are presented in Brès \& Colonius (2007).

\subsection{Validation}

The implementation of the numerical methods, the grid convergence, and boundary treatments were successfully validated following the procedures described by Rowley 


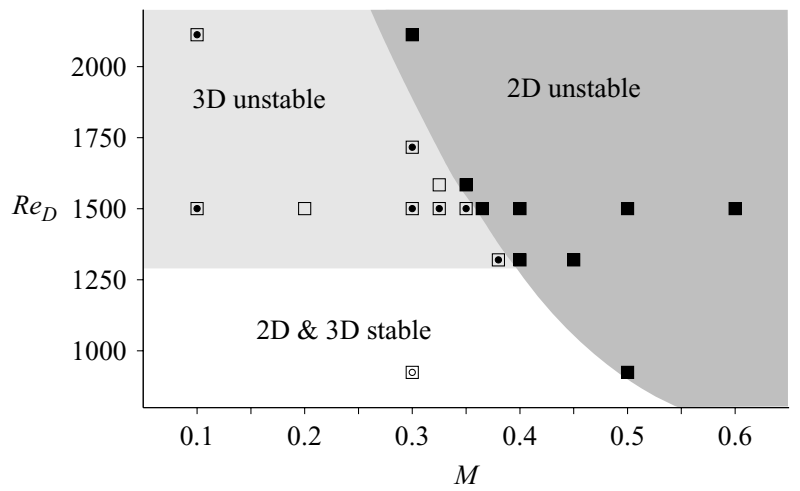

FIGURE 2. Schematic of the neutral stability curve for the cavity run series $2 \mathrm{M}(L / D=2$, $L / \theta_{0}=52.8$ ). Results from two-dimensional nonlinear simulations: two-dimensional stable ( $\square$ ) and unstable (ם); Results from three-dimensional linear analysis: three-dimensional stable (o) and unstable (๑). The different shaded regions indicate the approximate stability transitions. The critical conditions are estimated by linear interpolation between stable and unstable conditions. Details of each run are given in the Appendix.

et al. (2002). For brevity, we do not report the results here. The reader is referred to Brès (2007) for details. To validate the linear stability results, a direct approach was also considered (e.g. Brès 2007), where the eigenmodes are directly searched for using an Arnoldi method developed in the ARPACK software (e.g. Lehoucq et al. 2007), rather than isolated through long-time integration. In practice, the use of ARPACK was significantly limited by the size and complexity of our problem. The software was therefore only used to validate our time-domain methods. As expected, the dominant eigenmode and corresponding eigenvalue computed with ARPACK for the same test case were in excellent agreement with the results of the linear stability analysis.

\section{Linear stability results}

As described in the previous section, the three-dimensional linear stability analysis relies on the existence of subcritical conditions with a steady two-dimensional basic flow $\overline{\boldsymbol{q}}(x, y)$, an exact solution of the two-dimensional NS equations. However, for most experiments and realistic flight conditions, the flow parameters would be such that Rossiter modes do occur and eventually saturate into a periodically oscillating flow. Also, it must be acknowledged that the presence of three-dimensional instabilities is likely to alter the two-dimensional basic flow on which the present linear analysis is based. With this in mind, our approach here is to investigate the three-dimensional linear stability of a given base flow, regardless of potential interactions. Such an approach has been widely use to predict the stability and growth rate of boundary layers, for instance. As discussed in $\S 5$ and $\S 6$, the features observed in the linear results are in fact relevant to full nonlinear simulations and experiments.

The stability analysis is conducted as follows: given a cavity configuration and different flow conditions, several two-dimensional simulations are performed to construct the estimated neutral stability curve for the two-dimensional instabilities of the basic cavity flow (e.g. figure 2). The neutral stability curves are the starting point of the three-dimensional stability analysis: the goal here is to investigate whether threedimensional instability takes place before the onset of two-dimensional instabilities. For subcritical cases, the two-dimensional steady flow $\bar{q}$ is then extracted from the 
(a)

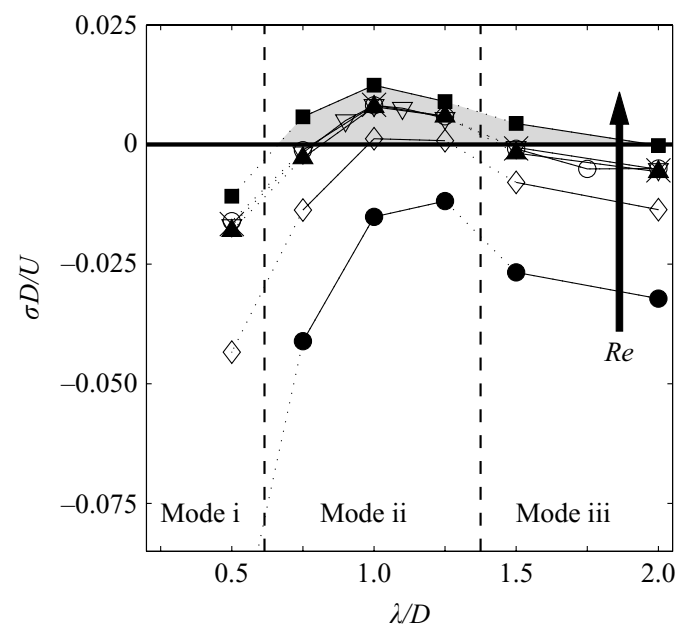

(b)

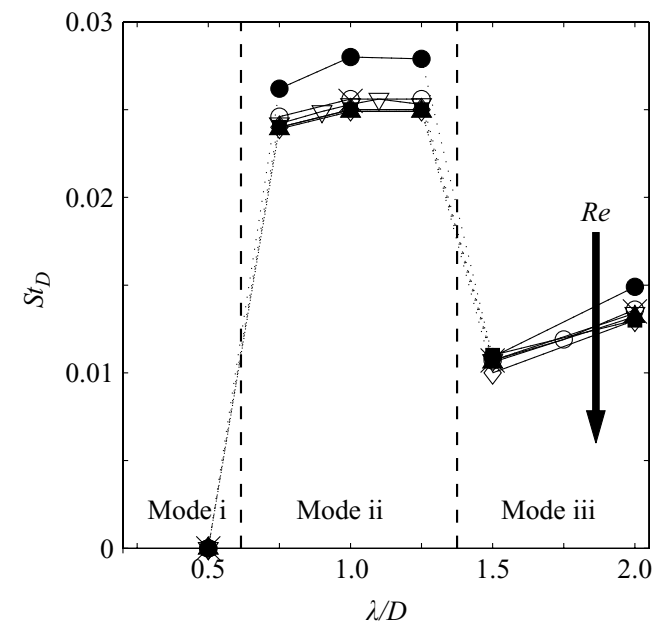

FIGURE 3. Three-dimensional linear stability results for run series $2 \mathrm{M}\left(L / D=2, L / \theta_{0}=52.8\right)$ as a function of the spanwise wavelength $\lambda / D$, for increasing Reynolds number (as indicated by the arrow) and different Mach numbers: $0.1<M<0.38$ for runs 2M01 $(\times)$, 2M0325 $(\triangle)$, 2M035 $(\bigcirc)$ and 2M038Re50 $(\diamond) ; M=0.3$ for runs 2M03Re35 $(\bullet), 2$ M03 $(\mathbf{\Delta})$ and 2M03Re65 (ద). The thick solid line represents the stability transition $\sigma_{D}=0$ and the region of positive growth rate is shaded. Different modes of instability are suggested. (a) Growth/damping rate; (b) frequency.

DNS and used as base flow for the linear three-dimensional simulations. As initial condition, a perturbation of given wavelength $\lambda$ (therefore looking at one $\beta$-mode at a time) is added to $\bar{q}$ and the three-dimensional linearized Navier-Stokes equations are solved. The least damped (or most unstable) eigenmode (e.g. figure 6) and the corresponding eigenvalue $\Omega=\omega+\mathrm{i} \sigma$ are then determined from the long-time response of the cavity. The non-dimensionalized growth/damping rate $\sigma D / U$ and Strouhal number $S t_{D}=\omega D / 2 \pi U$ are computed in each case for a set of discrete spanwise wavelengths (e.g. figure 3) and the stability of the three-dimensional mode is reported on the stability curve. The parameters for the different runs, as well as the results from the stability analysis, are tabulated in the Appendix A.

\subsection{Mode properties}

Figures 3, 4 and 5 show the growth/damping rate and frequency of the dominant three-dimensional mode as a function of the spanwise wavelength, for different cavity configurations and flow conditions. The plots in these figures are representative of the results for the cavities of aspect ratio $L / D=1,2$ and 4 considered here. Different modes of instability, referred to as modes i, ii, and iii, can be identified. For a band of spanwise wavelengths around the size of the cavity depth $(\lambda / D \approx 1)$, the dominant mode has a positive growth rate under certain conditions. This most unstable mode (designated as mode ii) is unsteady and the oscillation frequency based on the cavity depth $D$ is comparable in all cases. This suggests that $D$, rather than $L$ or $\theta_{0}$, is the most appropriate length scale to characterize the three-dimensional instability. By contrast, the two-dimensional unstable Rossiter mode has frequency $f L / U$ scaling with the cavity length: in this feedback process, the resonant frequencies are directly connected to the times for vortical structures and radiated sound to travel downstream and upstream, respectively, across a cavity of length $L$. For the three-dimensional 
(a)

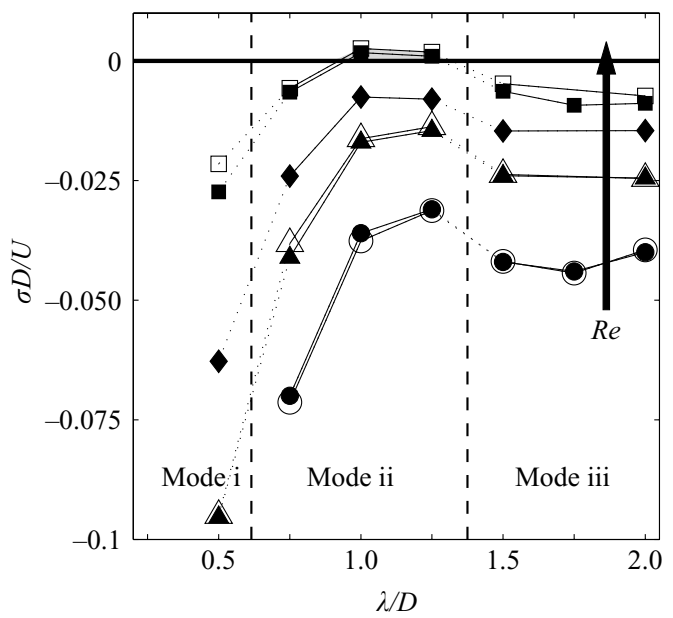

(b)

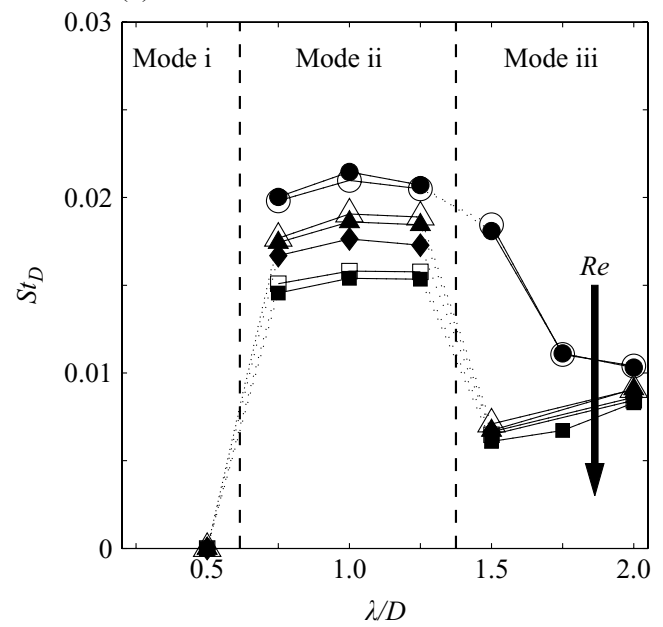

FIGURE 4. Three-dimensional linear stability results for run series TK2M $\left(L / D=2, L / \theta_{0}=30.1\right)$ as a function of the spanwise wavelength $\lambda / D$, for increasing Reynolds number (as indicated by the arrow). Two sets of Mach numbers are considered: $M=0.6$ for runs TK2M06 $(\bigcirc)$,

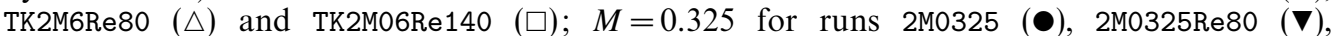
2 M0325Re100 ( ) and 2M0325Re140 (四). The thick solid line represents the stability transition $\sigma_{D}=0$ and the region of positive growth rate is shaded. Different modes of instability are suggested. (a) Growth/damping rate; $(b)$ frequency.

(a)

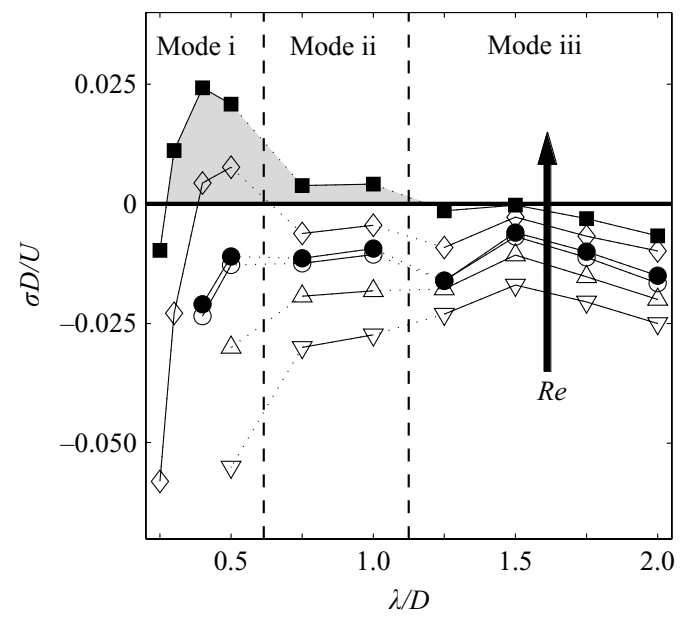

(b)

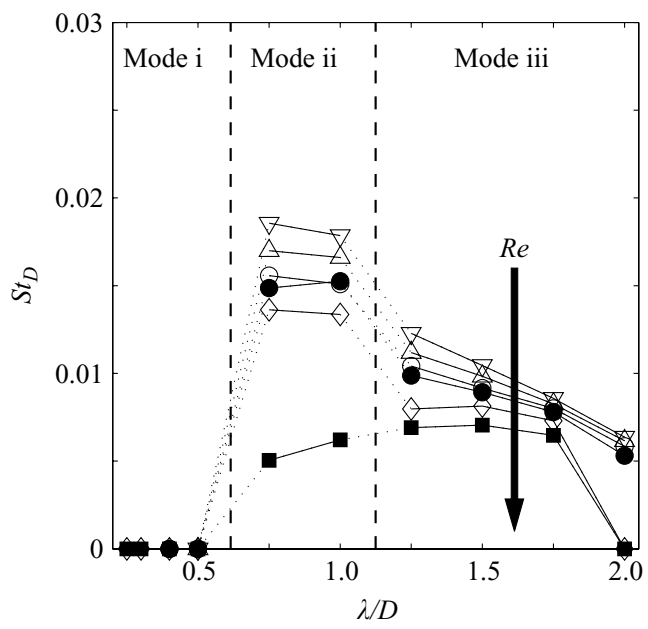

FIgURE 5. Three-dimensional linear stability results for run series $\mathrm{H} 1\left(L / D=1, L / \theta_{0}=23.2\right)$ as a function of the spanwise wavelength $\lambda / D$, for increasing Reynolds number (as indicated by the arrow). Two sets of Mach numbers are considered: $M=0.6$ for runs H1 $(\nabla)$, H1Re110 $(\triangle)$,

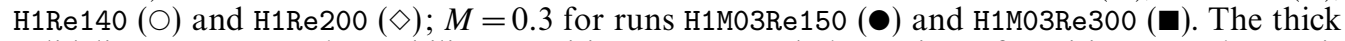
solid line represents the stability transition $\sigma_{D}=0$ and the region of positive growth rate is shaded. Different modes of instability are suggested. (a) Growth/damping rate; $(b)$ frequency.

instabilities, the frequency is found to be strongly associated with the two-dimensional base flow and its recirculating vortical flow within the cavity (also commonly referred to as the primary vortex), which is approximately of dimension $D$ in all cases. Details about the recirculating flow and the mode frequency are further discussed in $\S 3.3$. 
Aside from this oscillatory mode, the presence of other three-dimensional modes is suggested in figures 3, 4, and 5. In particular, the linear stability of the shortest cavity $L / D=1$ differs from the other cases. As the Reynolds number is increased, the first mode to become unstable is steady $\left(S t_{D}=0\right)$ and has a smaller spanwise wavelength $(\lambda / D \approx 0.5)$. A similar steady mode (referred to as mode i) is observed for cavities of larger aspect ratio but is not amplified. We argue that the specific properties of the three-dimensional mode for the square cavity are related to the recirculating vortical flow that occupies the whole cavity in that particular configuration (see details in $\S 4$ ).

Finally, the linear stability results also suggest the presence of another unsteady mode of larger spanwise wavelength $\lambda / D \geqslant 1$. . However, this mode iii does not have the largest linear growth rate at any of the conditions considered here, and is not observed in the three-dimensional nonlinear simulations we performed.

\subsection{Parameter dependence}

As mentioned previously, the parameters $L / D, L / \theta_{0}, R e_{D}$ and $M$ control the onset of the shear-layer (Rossiter) oscillation and therefore whether a steady two-dimensional flow exists. Within the domain of two-dimensional stability, our linear results show that the flow parameters affect the properties of the three-dimensional modes in four respects. First, as discussed above, the cavity aspect ratio controls which mode (namely i or ii) is the dominant mode; secondly, the Reynolds number has a direct effect on the growth rate as viscosity plays a stabilizing role; thirdly, a change in certain parameters (e.g. $L / \theta_{0}$ and $R e$ ) modifies the strength of the recirculating region in the two-dimensional base flow and indirectly the mode growth rate and frequency; and finally, the Mach number has little influence on the mode properties.

\subsubsection{Reynolds number}

As indicated by the arrows in figures 3, 4 and 5, an increase in Reynolds number has two effects on the properties of the instability: it significantly increases the growth rate and moderately decreases the oscillation frequency. To fully ascertain the effect of $R e$ on the onset of three-dimensional modes, we performed a set of numerical experiments in which the base flow was artificially held constant as $R e$ was increased (for details, see Brès \& Colonius 2007). The results confirmed that the growth rate of the dominant mode is directly driven by the Reynolds number. Viscosity damps the instability and there is a critical Reynolds number, above which the flow becomes three-dimensionally unstable. Since the Reynolds numbers considered in this study are low, the three-dimensional unstable modes are likely to exist for high-Reynoldsnumbers flows in practical applications.

\subsubsection{Boundary layer thickness}

The results show that the parameter $L / \theta_{0}$ controls whether the base flow permits three-dimensional instability, and has an impact on the oscillation frequency. A closer inspection of the three-dimensional linearized Navier-Stokes equations reveals that the influence of this parameter is limited to its effect on the base flow. Our parametric study indicates that when the initial boundary layer momentum thickness is reduced while keeping the other parameters $R_{D}, M$ and $L / D$ constant, the strength of the recirculating region in the two-dimensional base flow increases, and three-dimensional instabilities eventually develop. These observations suggest again that a critical strength of recirculating flow in the two-dimensional steady base flow needs to be reached for the presence of three-dimensional instability. 
(a)

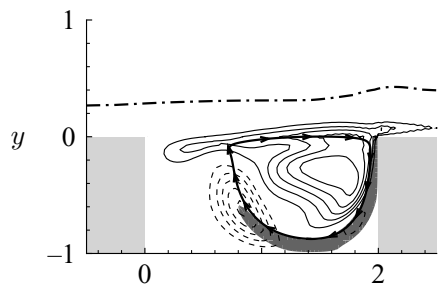

(d)

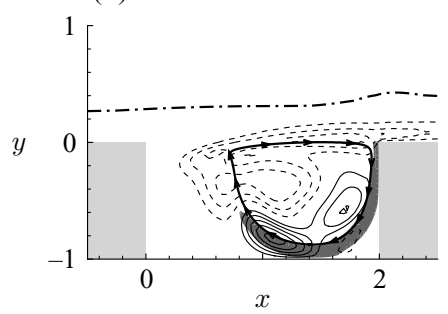

(b)

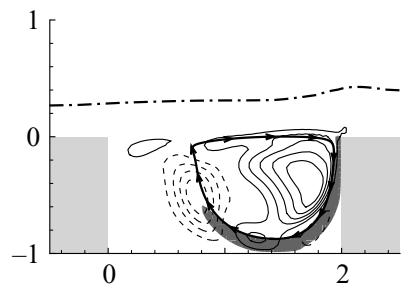

(e)

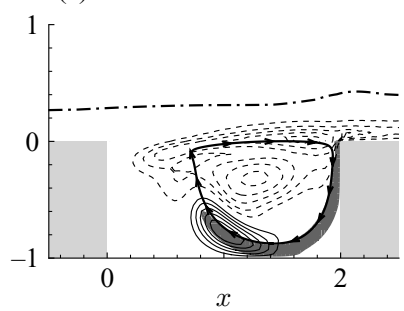

(c)
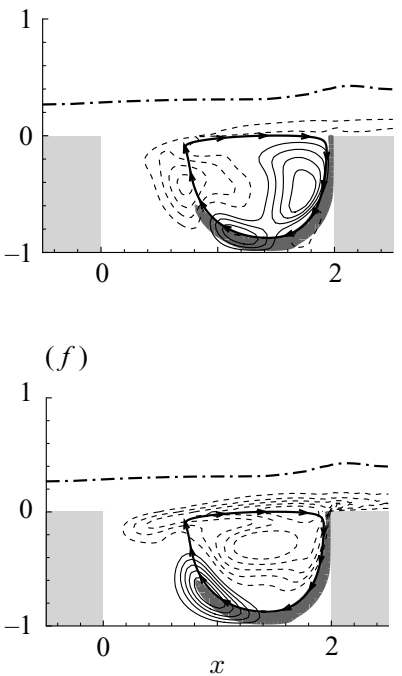

FiguRE 6. Contours of the linearized perturbation velocity $u^{\prime} / U$ for the unstable eigenfunction of spanwise wavelength $\lambda / D=1$ for run 2M0325. Six snapshots $(a-f)$, corresponding to half a time period of the instability, are shown. The velocity levels are arbitrary and the negative contours are dashed. The dark grey shading indicates the zone of potential centrifugal instability discussed in $\S 4$. Other features of the two-dimensional base flow: $\rightarrow$, streamline; - - shear-layer thickness.

\subsubsection{Mach number}

From figures 3 and 4 , it is clear that properties of the dominant three-dimensional mode are essentially independent of the Mach number, for subcritical conditions and subsonic speed up to 0.6. A set a numerical experiments confirmed this result (for details, see Brès \& Colonius 2007). This is a substantially different behaviour than the Rossiter modes. It indicates that the instability is unrelated to acoustic waves and other effects of compressibility. It is also consistent with the observation that the three-dimensional instability is active in the recirculating region within the cavity where the flow speed is much lower than the free-stream value.

As a result, the critical conditions for the onset of the three-dimensional instability are expected to be nearly independent of the Mach number, at least up to $M=0.6$. In figure 2, the critical Reynolds number $R e_{D} \approx 1300$ is estimated at $M=0.3$ from the run series 2M03, and is assumed to remain constant for the range of Mach number $0<M<0.4$, up to the limit of two-dimensional stability.

These results also suggest that the proper scaling for the velocity may not be the free-stream velocity, but rather some characteristic velocity inside the cavity. Alternative scalings are considered in $\S 4.2$.

\subsection{Eigenmode structure}

The unstable eigenfunction of wavelength $\lambda / D=1$ for run 2M0325 is depicted in figure 6 and is representative of all the three-dimensional unsteady instabilities. A cycle of growth and decay of the disturbance can be observed, as it rotates around the primary vortex of the two-dimensional base flow in the downstream half of the cavity. Starting from the cavity trailing edge, a zone of strong growth exists near the downstream wall where the shear layer impinges on the cavity corner. As the fluid in the cavity recirculates upstream and towards the bottom of the cavity, the 
disturbance amplitude decays slightly, before growing again when moving away from the bottom wall and toward the shear layer. In the shear layer, the perturbation is then damped and convected downstream, until it reaches the cavity corner and starts this cycle again. Overall, a larger growth rate than decay rate in this process leads to reinforcement of the disturbance and ultimately instability.

Another distinctive characteristic of the three-dimensional instability is that the oscillation frequency is about an order of magnitude smaller than the typical frequency of the two-dimensional shear-layer (Rossiter) mode. A detailed inspection of the base flow properties shows that the mode frequency is related to the closed streamlines in the primary vortex. The time for a disturbance to advect around the recirculating flow is computed for a discrete set of streamlines and the corresponding non-dimensionalized frequencies are compared to the instability frequency from the linear stability analysis. Under most conditions, there is a region in the primary vortex where the frequency computed from the streamline matches the mode frequency. Such a streamline is presented in figure 6 and shows good agreement with the motion of the disturbances. The smaller average velocity along these streamlines (typically $5 \%$ to $10 \%$ of the free-stream velocity, as shown below in figure $8 b$ ) also accounts for the low frequency measured.

This feature of the base flow also sheds light on the dependence of the predicted instability frequency on the flow parameters. In figures 3, 4 and 5, similar trends are observed when the base flow is modified through an increase of the Reynolds number or of the incoming boundary layer thickness: that is, the oscillation frequency decreases for higher $R e$ or smaller $L / \theta_{0}$. In all of these cases, the strength of the recirculating region in the cavity decreases, so the travel time of disturbances in the primary vortex increases, leading to higher three-dimensional mode frequencies. We note that this effect is moderate however and it is not expected that the oscillation frequency would be significantly altered at higher $R e$. In the range of conditions considered, the properties of the two-dimensional steady and time-averaged base flow for sub- and supercritical cases are comparable.

\section{Instability mechanism}

\subsection{Centrifugal instability}

We now address the mechanism of three-dimensional instability. The linear results indicate that the instability depends on the strength of the recirculating region in the downstream half of the cavity. We argue that the instability mechanism is a generic centrifugal instability associated with the closed streamlines in the recirculating vortical flow near the downstream cavity wall.

The centrifugal instability problem was first considered for swirling inviscid flow by Rayleigh, who derived a circulation criterion for stability (e.g. Drazin \& Reid 1981). The classical theory for circular streamlines was generalized to two-dimensional inviscid flow by Bayly (1988). For the centrifugal instability to occur, a sufficient condition is that the magnitude of the angular velocity decreases outward in some region of the flow with closed streamlines. Barkley, Gomes \& Henderson (2002) applied this theory to three-dimensional instability in the flow over a backward-facing step. Following their notation, the Rayleigh discriminant is defined as

$$
\eta(x, y)=-\frac{\partial\|\boldsymbol{r}(x, y) \times \overline{\boldsymbol{u}}(x, y)\|^{2}}{\partial r},
$$


(a) $y$

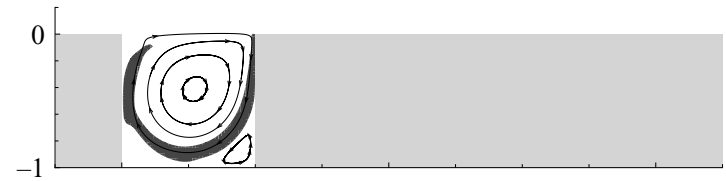

(b) $y$

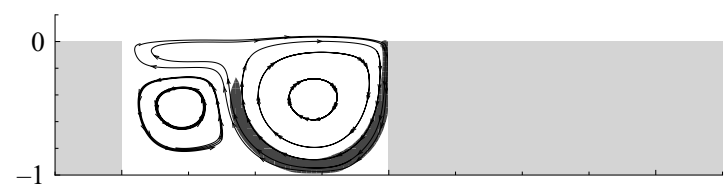

(c) $y$

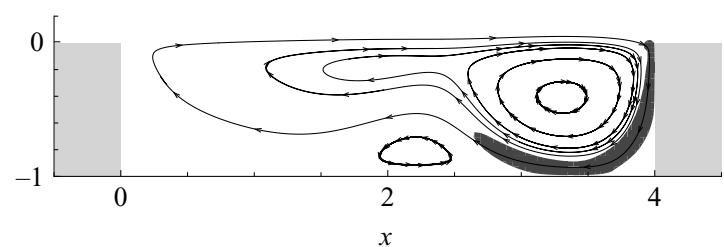

FiguRE 7. Streamlines and Rayleigh discriminant of the two-dimensional steady (or time-averaged) base flow. The dark grey shading indicates where $\eta$ is greater than $5 \%$ of its maximum value. $(a)$ Run H1Re200; (b) run 2M06; $(c)$ run TK4M03Re65. Cases $(a),(c)$ are two-dimensional subcritical and $(b)$ is supercritical.

where $\boldsymbol{r}(x, y)=\left(x-x_{c}, y-y_{c}\right),\left(x_{c}, y_{c}\right)$ is the centre about which the angular velocity is defined, and $r=\|\boldsymbol{r}(x, y)\|$. In practice, the centre $\left(x_{c}, y_{c}\right)$ was chosen to be the approximate location of the minimum velocity in the recirculating region inside the cavity. The discriminant $\eta$ is weakly dependent on the choice of the centre of rotation: computations with $\pm 10 \%$ error on $\left(x_{c}, y_{c}\right)$ lead to similar results. The flow is centrifugally unstable in the inviscid limit where $\eta>0$.

Figure 7 shows the Rayleigh discriminant of the two-dimensional steady (or timeaveraged) base flow for different cavity configurations. The contours represent the region where $\eta$ is greater than $5 \%$ of its maximum value. Additionally, the streamlines are plotted to visualize the flow. The zone where the angular velocity decreases radially corresponds the downstream part of the recirculating flow near the cavity wall. It also corresponds to the region of maximum growth of the disturbance, as discussed in the previous section. The fluid in the recirculating flow experiences a regime similar to solid-body rotation, with velocity and circulation along the streamlines increasing linearly with the distance to the centre of rotation (see figure 8). Intuitively, the presence of the downstream and bottom walls forces a rapid decrease in momentum at the outer streamlines. Because of the wall, the fluid is driven in the spanwise direction to form cellular patterns. As the walls are an intrinsic part of all configurations, any cavity flow should be inherently unstable because of the centrifugal instability mechanism, and our simulations do show regions of positive Rayleigh discriminant even for three-dimensional stable cases. However, the centrifugal stability criterion is only a sufficient condition for inviscid flow. As the parametric study of the Reynolds number effect showed, viscosity plays a stabilizing role. In general, simulations with a higher $R e$ lead to larger values and broader zones of positive Rayleigh discriminant. This result is consistent with the higher linear growth rate of the instability measured in these cases.

Likewise, two-dimensional time-averaged base flows at supercritical conditions exhibit similar regions of potential centrifugal instability. Comparison of the Rayleigh discriminant between the subcritical run 2M0325 in figure 6 and the supercritical run 
2M06 in figure 7(b) shows comparable results, with a slight increase in the levels and extent of the instability zone in the latter case. This observation suggests again that the centrifugal instability mechanism is relevant for cavity flow vortices (in a time-averaged sense) at higher Reynolds numbers.

As mentioned previously, cavities with aspect ratio $L / D=1$ feature slightly different characteristics. The recirculating vortical flow now occupies the whole cavity and the motion of disturbances in that vortex is affected not only by the downstream and bottom walls, but by the upstream wall as well. The computation of the Rayleigh discriminant reflects these distinctive features. It is clear in figure $7(a)$ that the region of potential centrifugal instability is much larger, covering the outward streamlines of the primary vortex from the trailing edge of the downstream wall all the way back to the shear layer. The influence of the upstream wall is believed to be the main explanation for the differences in the instability spanwise wavelength and oscillation frequency observed between cavities of smaller and larger aspect ratio.

\subsection{Connection with other centrifugal instabilities}

While this is the first time, to our knowledge, that three-dimensionality connected to centrifugal instabilities has been reported for open cavity flows, past studies of global instabilities have revealed centrifugal instabilities for related flow configurations. Most of these studies focused on critical flow conditions for the onset of centrifugal instability and the properties of the resulting three-dimensional mode.

\subsubsection{Flow past a backward-facing step}

Barkley et al. (2002) considered incompressible flow over an spanwise homogeneous backward-facing step geometry, which can be related to that of a wide cavity at appropriate parameter ranges. The run series TK4M03 for our longest cavity of aspect ratio $L / D=4$ at low Mach number $M=0.3$ was set up to match the range of Reynolds number and the laminar boundary layer momentum thickness of the step simulations. Barkley et al. (2002) discovered the existence of amplified largescale spanwise-periodic vortical structures in this flow. They argued that the source of the three-dimensionality was the centrifugal instability mechanism within the closed recirculation bubble formed between the lip and the downstream floor of the backward-facing step. Much like our linear stability analysis of cavity flow, they showed that the flow was linearly unstable to three-dimensional disturbances over a finite range of spanwise wavelengths. The reported critical Reynolds number for the instability $R e_{h}=U h / v=748$ is of the same order as $R e_{D}=960$ for the run series TK4M03 (here $h$ is the step height similar to the cavity depth $D$ ). However, their critical three-dimensional mode is steady with a spanwise wavelength $\lambda / h=6.9$ (see table 1 ). As the three-dimensionality in both cases is believed to arise from the recirculating flows in the step separation zone and downstream half of the cavity, a possible source of these discrepancies is the differences between the closed streamlines in both flows. The streamlines in the step separation zone in Barkley et al.'s (2002) simulations are elliptical, rather than circular as in the cavity flows.

\subsubsection{Lid-driven cavity flows}

The lid-driven cavity (LDC) also features three-dimensional centrifugal instabilities (e.g. Albensoeder et al. 2001). While cavity flows exhibit a much richer variety of fluid dynamic processes (shear-layer instabilities, vortex-surface interaction, acoustic wave propagation) compared to traditional incompressible bounded lid-driven flows, the two-dimensional steady base flows obtained from simulations of subcritical cavity flows are similar to the corresponding LDC basic flow. Numerous representations 


\begin{tabular}{|c|c|c|c|c|c|c|c|c|}
\hline & $L / D=1$ & $\lambda / D$ & $R e$ & $\operatorname{Re}_{l i d}$ & $\operatorname{Re}_{C}$ & $S t_{D}$ & $S t_{\text {lid }}$ & $S t_{C}$ \\
\hline \multirow[t]{3}{*}{ LDC } & Albensoeder et al. (2001) & 0.408 & - & 786 & - & - & 0 & - \\
\hline & Albensoeder et al. $(2001) \dagger$ & 0.398 & - & 810 & - & - & 0 & - \\
\hline & Theofilis et al. (2004) & 0.409 & - & 782 & - & - & 0 & - \\
\hline Couette & DiPrima et al. (1984) & 0.46 & - & - & 317 & - & - & 0 \\
\hline \multirow[t]{2}{*}{$\mathrm{OC}$} & run series $\mathrm{H} 1$ & 0.5 & 4120 & 382 & 209 & 0 & 0 & 0 \\
\hline & run series H1M03 & 0.4 & 4060 & 377 & 208 & 0 & 0 & 0 \\
\hline \multirow[t]{5}{*}{ LDC } & Aidun et al. (1991)†† & $\approx 1$ & - & 875 & - & - & 0.111 & - \\
\hline & Ramanan \& Homsy (1994) & 1.047 & - & 730 & - & - & 0.100 & - \\
\hline & Ding \& Kawahara (1999) & 0.849 & - & 920 & - & - & 0.079 & - \\
\hline & Albensoeder et al. (2001) & 0.848 & - & 933 & - & - & 0.078 & - \\
\hline & Theofilis et al. (2004) & 0.849 & - & 922 & - & - & 0.079 & - \\
\hline \multirow[t]{2}{*}{$\mathrm{OC}$} & run series H1M03 & 1 & 5900 & 452 & 277 & 0.010 & 0.130 & 0.213 \\
\hline & $L / D=2$ & & & & & & & \\
\hline \multirow[t]{2}{*}{ LDC } & Albensoeder et al. (2001) & 1.182 & - & 353 & - & - & 0.092 & - \\
\hline & Theofilis et al. (2004) & 1.102 & - & 360 & - & - & 0.093 & - \\
\hline \multirow[t]{4}{*}{$\mathrm{OC}$} & run series $2 \mathrm{MO3}$ & 1 & 1300 & 200 & 144 & 0.026 & 0.169 & 0.235 \\
\hline & run series TK2M0325 & 1 & 2000 & 198 & 133 & 0.016 & 0.160 & 0.237 \\
\hline & run series TK2M06 & 1 & 1990 & 198 & 140 & 0.017 & 0.166 & 0.235 \\
\hline & $L / D=4$ & & & & & & & \\
\hline \multirow[t]{2}{*}{ LDC } & Albensoeder et al. (2001) & 1.220 & - & 288 & - & - & 0.091 & - \\
\hline & Theofilis et al. (2004) & 1.257 & - & 290 & - & - & 0.090 & - \\
\hline \multirow[t]{2}{*}{$\mathrm{OC}$} & run series $4 \mathrm{MO} 3$ & 1.25 & 2370 & 176 & 118 & 0.012 & 0.158 & 0.236 \\
\hline & run series TK4M03 & 1.25 & 960 & 146 & 110 & 0.026 & 0.172 & 0.229 \\
\hline BFS & Barkley et al. (2002) & 6.9 & 750 & - & - & 0 & - & - \\
\hline
\end{tabular}

TABLE 1. Critical conditions of three-dimensional centrifugal instability for flows over a backward-facing step (BFS), lid-driven cavity (LDC), Couette and open cavity (OC) flows for different aspect ratios. To enable direct comparison with our linear results for cavity flows, the critical parameters were linearly interpolated whenever conditions of both sides of the three-dimensional stability transition were available. Critical conditions for both steady and unsteady modes are reported. The Reynolds number and non-dimensionalized frequency are defined as $U_{*} D / v$ and $f D / U_{*}$ where different velocity scales $U_{*}$ are considered for the cavity flows: the free-stream velocity $U$, the average velocity $U_{\text {lid }}$ along the cavity at $y=0$ and the maximum average velocity $U_{C}$ along the closed streamlines of the two-dimensional base flow vortex, as defined in $\S 4.2 .3$. $\dagger$ Experimental results. $†$ Mode frequency estimated by Benson $\&$ Aidun (1992).

of the two-dimensional streamlines in lid-driven cavity configurations at different Reynolds numbers are available in the literature (see Ramanan \& Homsy 1994; Ding \& Kawahara 1999 for a square cavity, and Albensoeder, Kuhlmann \& Rath 2001 for cavity of aspect ratio 4). These results can be related to the basic cavity flows in figure 7. Because of the presence of a comparable recirculating region in both flows, similar trends can be expected for the stability of LDC and cavity flows. Several experimental and numerical studies have reported three-dimensional modes in square LDC flow and measured the corresponding critical conditions. Additionally, for lid-driven cavities of aspect ratio 1,2 and 4, detailed linear stability analysis were 
(a)

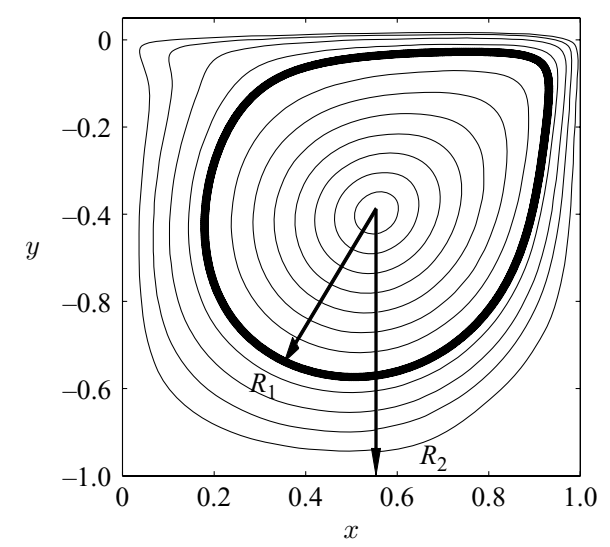

(b)

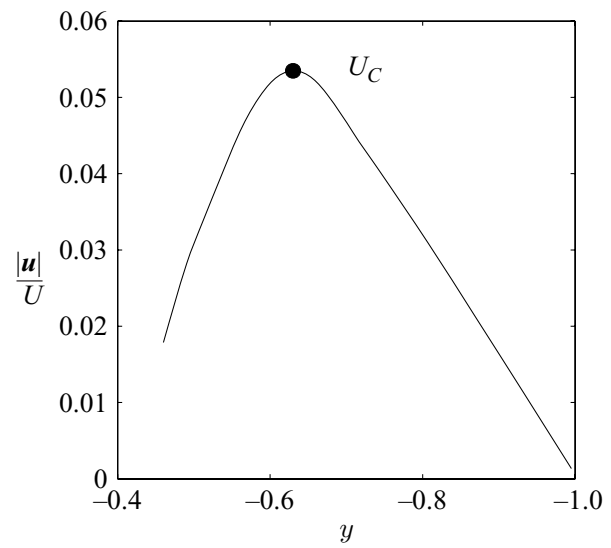

FIGURE 8. Properties of the two-dimensional steady flow in run H1Re140 for comparison with Couette flow. (a) Streamlines (only one streamline out of 5 is shown); $(b)$ magnitude of the average velocity $|\boldsymbol{u}| / U$ along the streamline going through $\left(x_{c}=0.55, y\right) ;(\bullet)$ end of the solid-body rotation regime. The corresponding Couette flow cylinder radiui $R_{1}$ and $R_{2}$, as well as the new velocity scale $U_{C}$, are shown.

conducted by Albensoeder et al. (2001) and Theofilis et al. (2004). All the results are presented in table 1.

However, since there is no direct correspondence between the Reynolds number $R e_{D}=U D / v$ defined for the cavity flow and $R e_{l i d}=U_{l i d} D / v$ for the lid-driven flow, comparisons remain qualitative. A different scaling than the free-stream velocity is considered here, to enable a closer comparison with LDC results. The average streamwise velocity along the cavity mouth at $y=0$ is computed for each twodimensional steady basic flow field, and is interpolated for the critical conditions. The critical Reynolds numbers and frequencies are then rescaled using that average velocity $U_{\text {lid }}$ and are shown in table 1 . While the traditional non-dimensionalization leads to a range of critical conditions strongly dependent on the cavity aspect ratio and the initial boundary layer momentum thickness, the rescaled results have the same order of magnitude, independently of the cavity configuration. The average velocity $U_{\text {lid }}$ is typically $5 \%$ to $15 \%$ of the free-stream velocity, which accounts for the lower critical Reynolds number $R e_{l i d}$ and higher corresponding frequency $S t_{l i d}$. There is also a closer agreement between the LDC and cavity flow results.

\subsubsection{Couette flows}

Another alternative scaling for the velocity is based on the observation that the recirculating vortical flow inside the cavity exhibits characteristics similar to a solid-body rotation away from the walls (as confirmed by PIV measurements from Chatellier, Laumonier \& Gervais 2006). This feature suggests that the cavity flow could be compared to the special case of Couette flows where the inner cylinder of radius $R_{1}$ rotates with constant angular velocity $\Omega_{1}$ while the outer cylinder of radius $R_{2}$ is at rest. This is particularly relevant for the square cavity, where the recirculating flow occupies the whole cavity and plays the role of the inner cylinder while the cavity walls correspond to the stationary outer cylinder. The stability of such a flow has been widely studied and is often mentioned as a classical example of centrifugal instabilities. 
DiPrima, Eagles \& Ng (1984) computed the critical values of the Reynolds number and corresponding axial wavenumber in Couette flow as a function of the radius ratio $R_{1} / R_{2}$ for axisymmetric stationary modes. Note that their results were made dimensionless by scaling length with the gap width $d=R_{2}-R_{1}$ and velocity with the inner cylinder velocity $\Omega_{1} R_{1}$. To enable comparison with the Couette flow stability results, closed streamlines inside the recirculating flow are extracted from our results and the average velocity along each streamline is computed. From figure $8(b)$, it is clear that the fluid in the vortex rotates with nearly constant angular velocity, as the average velocity along the inner streamlines is proportional to the distance from the centre of rotation, until the influence of the solid wall is felt, leading to the approximate definition of the convective velocity scale $U_{C}=\Omega_{1} R_{1}$. Note that the objective here is only to achieve qualitative comparison, as it is not possible to unambiguously define $R_{1}$ and $R_{2}$ for the cavity flow.

We estimated the radius ratio for run H1Re140, which is the last available condition from our linear stability analysis before the stationary mode of wavelength $\lambda=0.5$ becomes unstable. From DiPrima et al. (1984), the corresponding critical Reynolds number and wavenumber are 317 and 0.46 respectively, in units of $D$ and $U_{C}$. These values are comparable to the square cavity flow data in table 1, highlighting once more the centrifugal character of the three-dimensional instabilities. Similarly to the previous non-dimensionalization inspired by the LDC flows, the rescaled results for the different cavity configurations have the same order of magnitude.

The stability results for the LDC and Couette flow also shed light on the particular properties of the instability in cavities of aspect ratio 1, that is the critical conditions correspond to a stationary mode of smaller spanwise wavelength, about $0.5 \mathrm{D}$. Based on the Couette flow analogy, these results suggest that this stationary mode is amplified in the particular configuration where the recirculating region occupies the whole cavity and interacts with all the cavity walls. Hence the unstable non-oscillatory mode of wavelength 0.4 reported in our linear stability analysis for $L / D=1$ cavities, and not for cavities of larger aspect ratio.

Finally, while this analysis makes clear the role of centrifugal instability in cavity flows, we note that it is not predictive without access to the DNS steady flow from which $U_{l i d}, U_{C}, R_{1}$ and $R_{2}$ are estimated.

\section{Nonlinear simulations}

To further investigate these instabilities, full three-dimensional, nonlinear simulations are performed. Both subcritical (run 2M0325-3D) and supercritical conditions (runs 2M06-3D and H1Re300-3D) are considered. For the three-dimensional simulation, the steady (or time-averaged) basic state $\bar{q}(x, y)$ extracted from the twodimensional DNS data is perturbed by small disturbances of spanwise wavelength $\lambda / D=2$ and $\lambda / D=1$. The full NS equations are then numerically solved for a homogeneous three-dimensional cavity of spanwise extent $\Lambda / D=2$. As the linear stability results suggest that the spanwise wavelength of the dominant threedimensional mode is in the range $0.4 \leqslant \lambda / D \leqslant 1.25$, such a spanwise extent is expected to be sufficient to capture all the flow physics. For the cavity of aspect ratio $L / D=2$, the grid contains about seven and a half million grid points, with $(N x=120, N y=60$, $N z=128)$ points across the cavity in the streamwise, depth and spanwise directions, respectively. The grid size is reduced to about three million grid points, with $(N x=60$, $N y=60, N z=128$ ) for the cavity of smaller aspect ratio $L / D=1$. For validation 


\begin{tabular}{|c|c|c|c|c|c|c|}
\hline Run & \multicolumn{3}{|c|}{$\begin{array}{c}2 \mathrm{M} 0325 \\
2 \mathrm{D} \text { subcritical }\end{array}$} & \multicolumn{3}{|c|}{$\begin{array}{c}2 \mathrm{M} 06 \\
2 \mathrm{D} \text { supercritical }\end{array}$} \\
\hline $\begin{array}{l}L / D \\
R e_{D} \\
L / \theta_{0} \\
M\end{array}$ & & $\begin{array}{c}2 \\
1500 \\
52.3 \\
0.325\end{array}$ & & & $\begin{array}{c}2 \\
1500 \\
52.3 \\
0.6\end{array}$ & \\
\hline & $S t_{D}$ & Mode & $\lambda / D$ & $S t_{D}$ & Mode & $\lambda / D$ \\
\hline Rossiter prediction & $\begin{array}{l}0.181 \\
0.422\end{array}$ & $\begin{array}{l}\text { I } \\
\text { II }\end{array}$ & $\begin{array}{l}\infty \\
\infty\end{array}$ & $\begin{array}{l}0.160 \\
0.372\end{array}$ & $\begin{array}{l}\text { I } \\
\text { II }\end{array}$ & $\begin{array}{l}\infty \\
\infty\end{array}$ \\
\hline 2D DNS & $0.241 \dagger$ & I & $\infty$ & 0.204 & I & $\infty$ \\
\hline 3D linear stability & $\begin{array}{l}0.025 \\
0.240 \dagger\end{array}$ & $\begin{array}{l}\text { ii } \\
\text { I }\end{array}$ & $\begin{array}{c}1 \\
\infty\end{array}$ & & $\begin{array}{l}\text { n.a. } \\
\text { n.a. }\end{array}$ & \\
\hline 3D DNS & $\begin{array}{l}0.025 \\
0.240 \dagger\end{array}$ & $\begin{array}{l}\text { ii } \\
\text { I }\end{array}$ & $\begin{array}{c}1 \\
\infty\end{array}$ & $\begin{array}{l}0.026 \\
0.352\end{array}$ & $\begin{array}{l}\text { ii } \\
\text { II }\end{array}$ & $\begin{array}{c}1 \\
\infty\end{array}$ \\
\hline
\end{tabular}

TABLE 2. Comparison of the dominant mode prediction for two- and three-dimensional runs with $L / D=2$. Only the most energetic frequencies $S t_{D}=f D / U$ are presented, along with the spanwise wavelength $\lambda / D$ of the instability. The original values from Rossiter $(1 / \kappa=1.75$, $\alpha=0.25$ ) are used in equation (1.1). $\dagger$ For subcritical conditions, the Rossiter modes are damped but the oscillation frequency can still be measured from the early times. The linear stability results are not available (n.a.) for supercritical conditions.

purposes, case 2M0325-3D was also performed on a coarser mesh with different initial conditions, and lead to identical results.

\subsection{Subcritical conditions}

The first configuration considered is again the subcritical run 2M0325, for a cavity of aspect ratio $L / D=2$ at low Mach and Reynolds number $\left(M=0.325, \operatorname{Re}_{D}=1500\right.$, $\left.L / \theta_{0}=53\right)$. The two-dimensional simulation shows that the flow is initially oscillating in the first Rossiter mode with a frequency $S t_{D}=f D / U=0.241$. The flow is stable with exponentially decaying amplitude, and ultimately converges to a steady state. This result is confirmed by the stability analysis. By setting $\beta=0$ in equation (2.2), the linear stability of the steady base flow can be investigated for perturbations of spanwise wavelength $\lambda / D=\infty$ (i.e. two-dimensional perturbations). In that case, the linear growth/damping rate and frequency of the dominant Rossiter mode are recovered. For run 2M0325, the mode is damped and the frequency is $S t_{D}=0.240$ (see table 2).

Figure 9 shows the time-history of the velocity $v / U$ for both two- and threedimensional simulations at approximately the same location in the middle of the cavity. Initially, the three-dimensional flow oscillates at a frequency corresponding to the two-dimensional Rossiter mode. This frequency and its first harmonic are evident in the spectrum in figure 10. After a transition period, the two-dimensional modes decay while the three-dimensional mode grows and saturates. The final frequency of oscillation is $S t_{D}=0.025$, corresponding to the frequency of the most unstable three-dimensional mode from the linear stability analysis (see table 2).

A visualization of the flow structures over half a period is presented in figure 11 . As the shear-layer oscillations are damped and eventually die out, the three-dimensional instability associated with the centrifugal mechanism is the only unsteady feature remaining in the flow: the growth and decay of disturbances rotating around the 


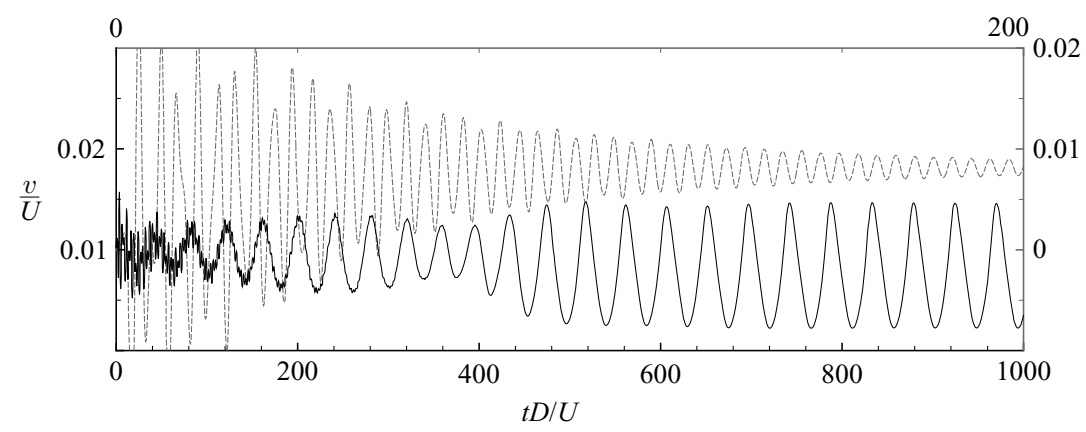

Figure 9. Time trace of normal velocity at $(x, y)=(0.5 L, 0)$ for two-dimensional run $2 \mathrm{M} 0325$ $(----)$ and three-dimensional run 2M0325-3D (- $)$ at $z=0$. The other flow variables exhibit the same characteristics. To show all the data clearly, the bottom and left axes correspond to the three-dimensional simulation, and the top and right axes to the two-dimensional run.

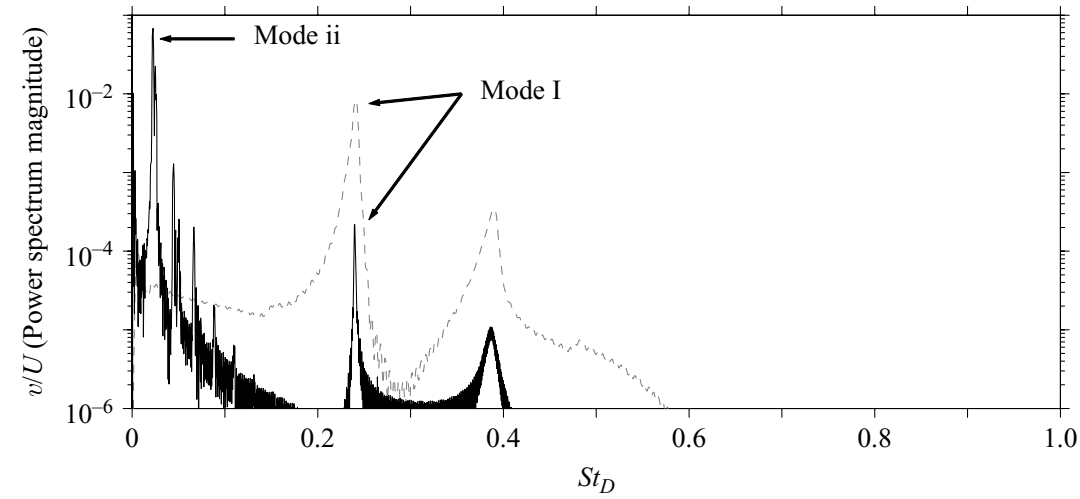

FIGURE 10. Spectrum of the normal velocity presented in figure 9; two-dimensional run 2M0325 $(----)$; three-dimensional run 2M0325-3D ( $(-)$. The different modes are identified and their harmonics can also be observed.

primary vortex can be observed in the cavity, as well as the formation of a cellular pattern similar to the linear stability results. As predicted, the spanwise wavelength of the three-dimensional instability is equal to one cavity depth. Spectral analysis in the spanwise direction shows that the energy spreads to all the other wavelengths (including harmonics) because of the nonlinear terms in the NS equations, but $\lambda / D=1$ remains the dominant wavelength.

Most of the three-dimensionality is confined to the internal flow inside the cavity, where the velocities remains small. The spanwise velocities in figure 11 correspond to only $1 \%$ of the free-stream velocity. The instantaneous maxima of $w / U$ are located near the downstream wall inside the cavity and reach about $2.5 \%$. Even smaller levels of spanwise velocity are mesured in the shear layer, which remains mostly twodimensional and stationary, despite the presence of the centrifugal instability. The shear-layer spreading rates for run 2M0325 and 2M0325-3D are shown in figure 14 below: both two- and three-dimensional simulations exhibit identical linear growth along the whole cavity. The shear-layer properties and the measurement of the spreading rate are discussed in more detail in $\S 5.2$.

Additionally, because the three-dimensional mode is oscillating about the mean flow, its contribution cancels out in a time-averaged sense: that is, the time-averaged flow 

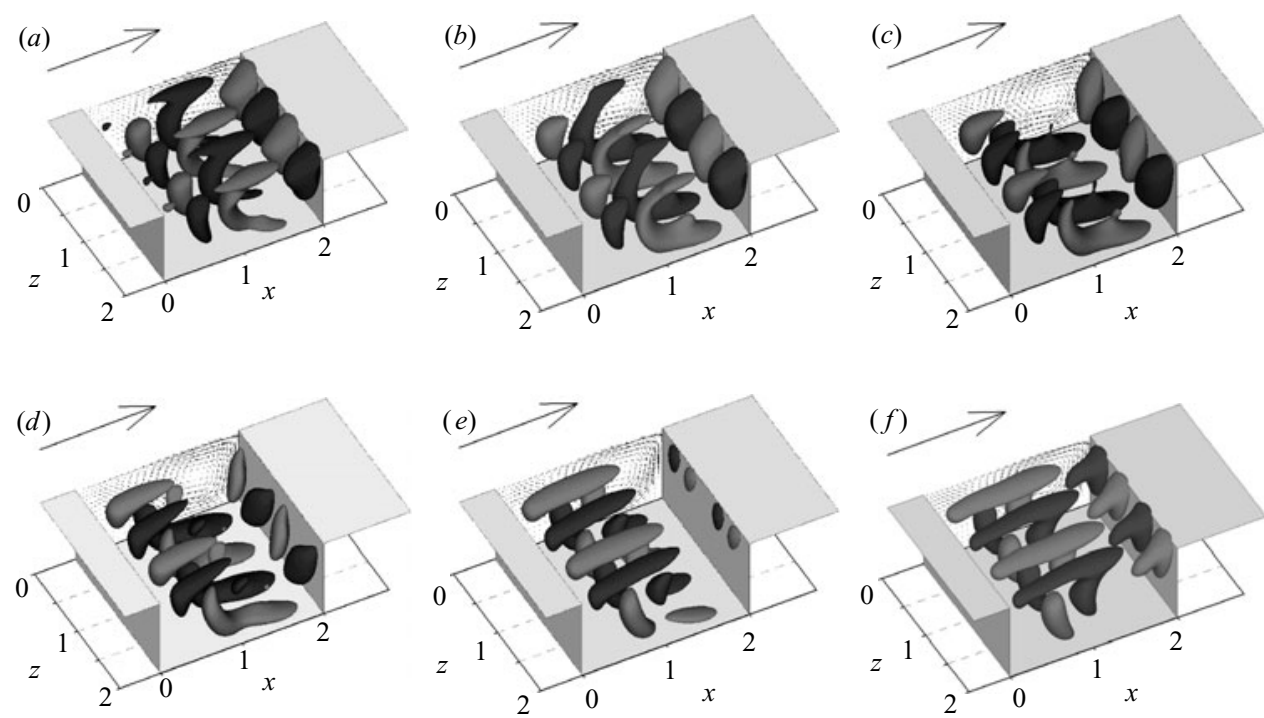

FIGURE 11. Visualization of the three-dimensional flow for run 2M0325-3D at six different times $(a-f)$ corresponding to approximately one-sixth phase intervals of a half-period of the three-dimensional instability. The iso-surfaces represent the spanwise velocity levels $w / U=-0.01$ and $w / U=0.01$. The whole spanwise extent of the cavity is shown and the wavelength $\lambda / D=1$ of the instability can clearly be observed. The velocity vectors in the streamwise cross-section at $z=0$ are shown inside the cavity and once in the free-stream for comparison.

does not exhibit the spanwise modulation of wavelength $\lambda / D=1$, and its spanwise average deviates from the two-dimensional predicted steady state by less than $2 \%$ of the free-stream quantities. Under the present conditions, the instability remains weak and is mainly active in the recirculating region within the cavity. The interaction with the shear layer is also weak and mostly limited to a low-frequency, small-amplitude oscillation.

\subsection{Supercritical conditions}

Supercritical conditions were obtained from the previous simulations by simply increasing the Mach number from $M=0.325$ to $M=0.6$ while keeping the other parameters constant. In run 2M06, the two-dimensional flow exhibits disturbances of growing amplitude and eventually saturates to a periodic oscillating flow of frequency corresponding to the Rossiter mode I. In this case, a time-averaged steady state $\bar{q}$ is extracted by averaging the periodic data and the nonlinear three-dimensional simulation is performed following the same procedure as the subcritical case.

The time-history of the velocity $u / U$ for runs 2M06 and 2M06-3D are compared in figure 12. Both Rossiter modes I and II are initially unstable in the two-dimensional simulation, but through a process of nonlinear amplification and saturation, mode I is selected, while mode II is damped and vanishes. In the three-dimensional simulation, after a transient exhibiting both Rossiter modes I and II, the self-sustained oscillations in the flow saturate into a periodic regime where the Rossiter mode II and the threedimensional instability can be observed simultaneously. The different mode frequencies are identified in figure 13 and reported in table 2. Much like the subcritical case 2M0325-3D, the flow exhibits three-dimensional structures of spanwise wavelength $\lambda / D=1$. 
(a)

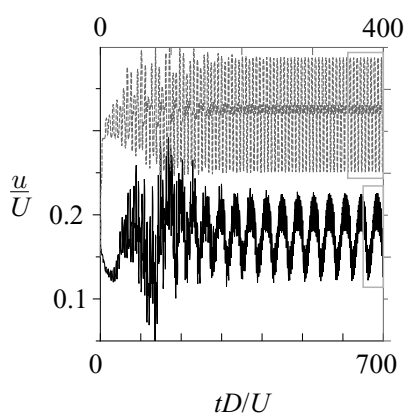

(b)

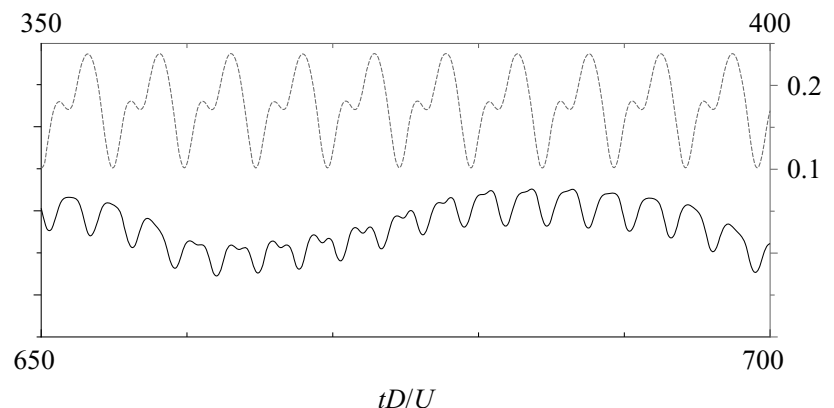

Figure 12. (a) Time trace of streamwise velocity at $(x, y)=(0.5 L, 0)$ for two-dimensional run 2M06 (-- - ) and three-dimensional run 2M06-3D ( $(-)$ at $z=0.25 D$. (b) Details of the signal in the dotted boxes on the right-hand side of $(a)$. To show all the data clearly, the bottom and left axes correspond to the three-dimensional simulation, and the top and right axes to the two-dimensional run.

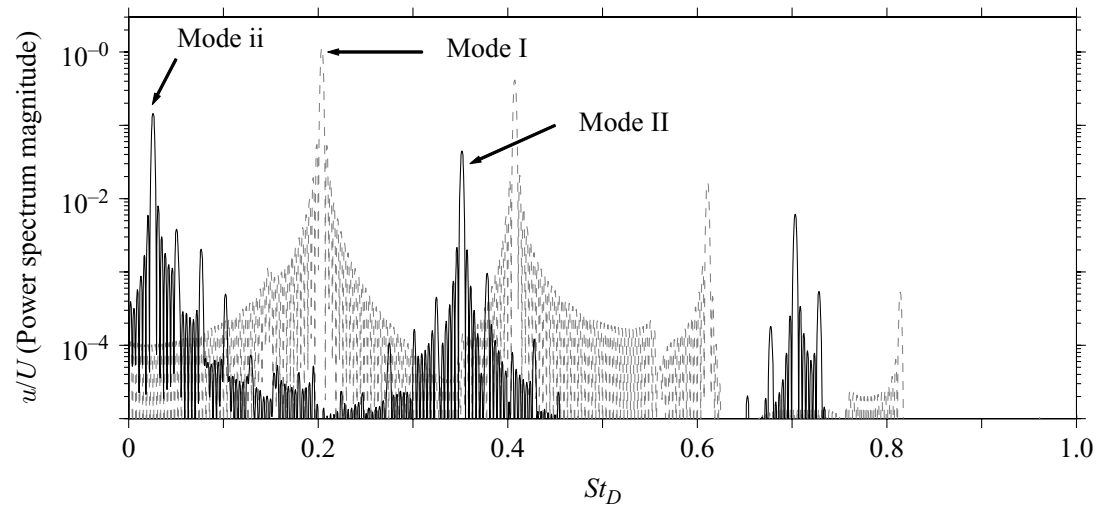

FIGURE 13. Spectrum of the streamwise velocity presented in figure $12(b)$; two-dimensional run 2M06 (----); three-dimensional run 2M06-3D (- - The different modes are identified and their harmonics can also be observed.

Since the two-dimensional flow is supercritical, linear results for run 2M06 are not available for direct comparison with the three-dimensional mode frequency observed here. However, the reported frequency and spanwise wavelength match the predicted result from the linear stability analysis of run 2M0325. There is no contradiction here, since we showed in $\$ 3.1$ that the Mach number has little influence on the characteristics of the three-dimensional mode. This result tends to indicate that linear results from subcritical cases (if such stable conditions exist) could deliver useful insight into the three-dimensional stability at higher Mach number, for corresponding supercritical conditions.

Another key feature is that the shear-layer oscillation frequency now corresponds to the Rossiter mode II, rather than mode I that is selected in strictly two-dimensional simulations. To better assess the instability properties of the shear layer, the vorticity thickness $\delta_{w}(x)=U /(\partial \bar{u}(x, y) / \partial y)_{\max }$ is computed, where $\bar{u}(x, y)$ is the time- and spanwise-averaged streamwise velocity. The vorticity thickness and its slope $\mathrm{d} \delta_{w} / \mathrm{d} x$ are typically used to measure the shear-layer spreading rate. Most researchers report that the shear layer over open cavities exhibits approximately linear growth, much 


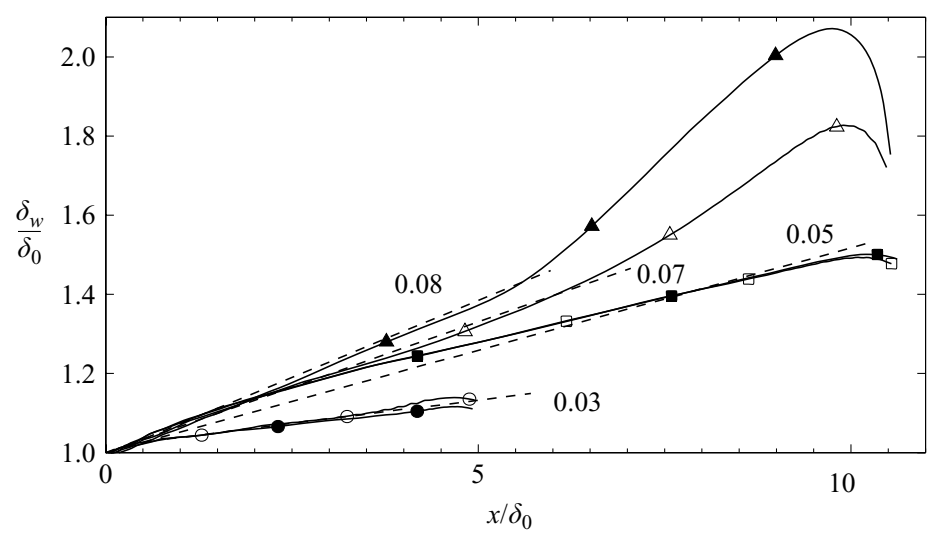

FigURE 14. Vorticity thickness $\delta_{w}$ along the shear layer and average slope $\mathrm{d} \delta_{w} / \mathrm{d} x(----)$. Cavity parameters: $L / D=1, R e_{D}=6960, L / \theta_{0}=23.2, M=0.6$ for runs H1Re300 (•) and H1Re300-3D (O); $L / D=2, R e_{D}=1500, L / \theta_{0}=52.8$ and $M=0.325$ for runs 2M0325 (- and 2M0325-3D ( $\square$ ), $M=0.6$ for runs 2M06 $(\mathbf{\Lambda})$ and 2M06-3D $(\triangle)$; For the latter two cases, data for $x / D>1$ were not considered in the calculation of $\mathrm{d} \delta_{w} / \mathrm{d} x$.

like free shear layers. However, the basic physics of these flows differs in two main aspects: the shear layer is subject to a strong acoustic feedback, and the presence the recirculating vortical flow in the downstream part of the cavity affects the entrainment and alters the shear-layer thickness.

The comparison of the spreading rates in figure 14 highlights these key features: for supercritical conditions (such as run 2M06-3D), the shear-layer oscillations lead to a higher initial spreading rate and a sharp increase around $x / \delta_{0} \approx 6$ (i.e. $x / D \approx 1$ ), which corresponds to the location of the recirculating region and the region of largeramplitude oscillations. In contrast, for the subcritical run 2M0325-3D with the same initial boundary layer momentum thickness, the spreading rate is approximately linear over the whole cavity and its value is about $25 \%$ lower. Also, the influence of the downstream wall is clearly shown for $x / \delta_{0}>10$ (i.e. $x / D>1.85$ ).

In both runs 2M06 and 2M06-3D, the shear layer initially exhibits linear growth for $x / \delta_{0}<6$, but there is a $15 \%$ decrease in the spreading rate between the twodimensional $\left(\mathrm{d} \delta_{w} / \mathrm{d} x \approx 0.08\right)$ and three-dimensional $\left(\mathrm{d} \delta_{w} / \mathrm{d} x \approx 0.07\right)$ simulations. These results are of the same order as the spreading rates measured in experiments (Sarohia 1975) and numerical simulations (Rowley et al. 2002; Larchevêque et al. 2003). Sarohia's measurement are based on the momentum thickness $\theta$ and can be related to vorticity thickness by $\delta_{w} \approx 4 \theta$ (exact equivalence for hyperbolic tangent profiles). As $L / \theta_{0}$ is increased from 52.5 to 105.2 , the spreading rate $\mathrm{d} \theta / \mathrm{d} x$ increases from 0.006 to 0.022 , and the cavity oscillation switches from mode I to mode II. Other experimental (e.g. Gharib \& Roshko 1987) and numerical (e.g. Rowley et al. 2002) studies on open cavity flows also report that larger shear-layer spreading rates are obtained as $L / \theta_{0}$ is increased, and ultimately lead to a higher dominant mode. In the present case, an increase in shear-layer spreading rate does not seem to be the cause of the higher mode observed in the three-dimensional simulation as the opposite trend can be observed in figure 14.

The shear-layer measurements are consistent with other observations in the flow field. As shown in figure 12, the oscillation amplitude of the limit cycle decreases between the two- and three-dimensional simulations. Likewise, a decrease in all the Reynolds stresses is observed, especially in the shear layer. Also, the sound 


\begin{tabular}{|c|c|c|c|c|c|c|}
\hline Run & \multicolumn{3}{|c|}{$\begin{array}{l}\text { H1M03Re300 } \\
\text { 2D subcritical }\end{array}$} & \multicolumn{3}{|c|}{$\begin{array}{c}\text { H1Re300 } \\
\text { 2D supercritical }\end{array}$} \\
\hline $\begin{array}{l}L / D \\
R e_{D} \\
L / \theta_{0} \\
M\end{array}$ & \multicolumn{2}{|r|}{$\begin{array}{c}1 \\
6960\end{array}$} & & & $\begin{array}{c}1 \\
6960 \\
23.2 \\
0.6\end{array}$ & \\
\hline & $S t_{D}$ & Mode & $\lambda / D$ & $S t_{D}$ & Mode & $\lambda / D$ \\
\hline Rossiter prediction & $\begin{array}{l}0.366 \\
0.854\end{array}$ & $\begin{array}{l}\text { I } \\
\text { II }\end{array}$ & $\begin{array}{l}\infty \\
\infty\end{array}$ & $\begin{array}{l}0.319 \\
0.745\end{array}$ & $\begin{array}{l}\text { I } \\
\text { II }\end{array}$ & $\begin{array}{l}\infty \\
\infty\end{array}$ \\
\hline 2D DNS & $0.357 \dagger$ & I & $\infty$ & 0.310 & I & $\infty$ \\
\hline 3D linear stability & $\begin{array}{c}0 \\
0.007 \\
0.361 \dagger\end{array}$ & $\begin{array}{l}\text { i } \\
\text { ii } \\
\text { I }\end{array}$ & $\begin{array}{c}0.4 \\
1 \\
\infty\end{array}$ & & $\begin{array}{l}\text { n.a. } \\
\text { n.a. } \\
\text { n.a. }\end{array}$ & \\
\hline 3D DNS & & comput & & $\begin{array}{c}0 \\
0.008 \\
0.307\end{array}$ & $\begin{array}{c}\text { i } \\
\text { ii } \\
\text { I }\end{array}$ & $\begin{array}{c}0.4 \\
0.4 \\
\infty\end{array}$ \\
\hline
\end{tabular}

TABLE 3. Comparison of the dominant mode prediction for two- and three-dimensional runs with $L / D=1$. $†$ For subcritical conditions, the Rossiter modes are damped but the oscillation frequency can still be measured from the early times. The linear stability results are not available (n.a.) for supercritical conditions.

pressure levels are lower overall for the three-dimensional simulation. This result is related to the decrease in oscillation amplitude previously mentioned. It is also in agreement with the general experimental observation that two-dimensional cavities are slightly louder than their three-dimensional counterparts. The weakened shear-layer coherence caused by turbulence is typically invoked to explain this trend. A similar argument can be made here, even at lower Reynolds number. As the three-dimensional centrifugal instability is established inside the cavity, the shear layer above develops the same spanwise fluctuations. The resulting reduced spanwise coherence of the vortical structures travelling downstream in the shear layer affects the receptivity of the cavity trailing edge, which, in turn, reduces the acoustic scattering, the leading-edge reinforcement of disturbances and the overall effectiveness of the feedback process.

In conclusion, our result suggests that both shear-layer and three-dimensional modes can coexist in the flow, and that their interaction seems to affect the selection of Rossiter modes.

\subsection{Multiple three-dimensional modes}

As mentioned in $\S 3.1$, the linear stability of the cavity of aspect ratio $L / D=1$ is different: the critical conditions correspond to the steady mode $i$ of smaller spanwise wavelength $(\lambda / D \approx 0.5)$. While the unstable oscillatory mode ii is still present at higher Reynolds number, it is less amplified than the steady mode for the conditions considered (see figure 5). From the linear analysis results, it is not possible to determine which mode (or modes) will be amplified in the nonlinear flow.

To investigate this question, the two-dimensional simulation H1Re300 is performed for a cavity of aspect ratio $L / D=1\left(M=0.6, R e_{D}=6960, L / \theta_{0}=23.2\right)$. This case corresponds again to supercritical conditions, as the two-dimensional flow oscillates in Rossiter mode I . The nonlinear three-dimensional simulation (referred to as run 
(a)

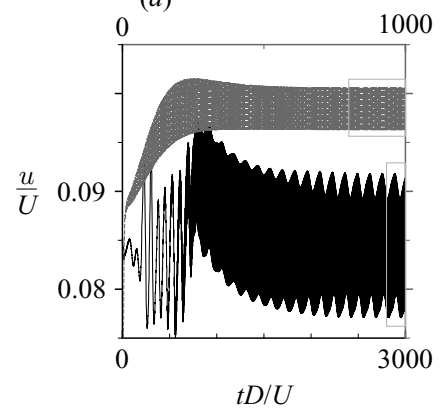

(b)

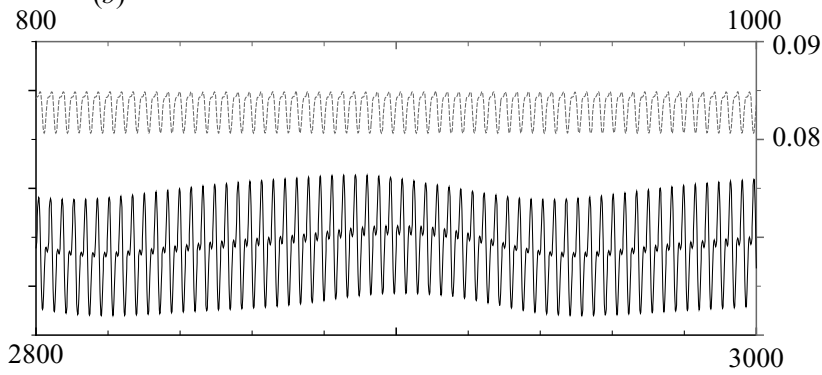

$t D / U$

Figure 15. (a) Time trace of streamwise velocity at $y / D=0$ and $x / D=0.5$ for two-dimensional run H1Re300 (----) and three-dimensional run H1Re300-3D ( - ) at $z / D=0$. (b) Details of the signal in the dotted boxes on the right-hand side of $(a)$. To show all the data clearly, the bottom and left axes correspond to the three-dimensional simulation, and the top and right axes to the two-dimensional run.

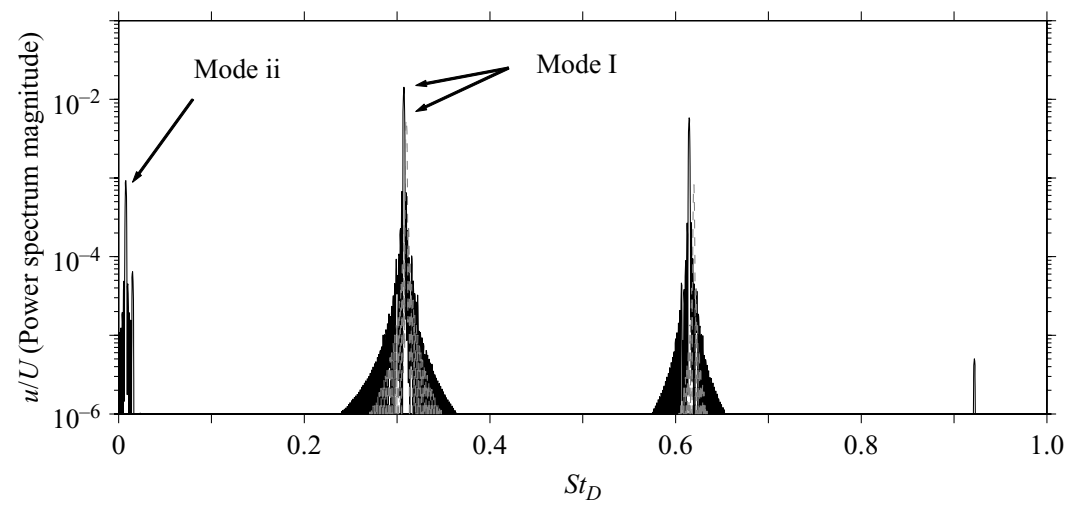

FIGURE 16. Spectrum of the streamwise velocity presented in figure $15(b)$; two-dimensional run H1Re300 (----); three-dimensional run H1RE300-3D (- - . The different modes are identified and their harmonics can also be observed.

H1Re300-3D) is then performed. According to the linear stability results in table 1, both modes $i$ and ii should be unstable, as $R e_{D}=6960$ is higher than their respective critical Reynolds numbers.

Figure 15 shows the time-history of the velocity $u / U$ for runs $H 1 \operatorname{Re} 300$ and H1Re300-3D. Unlike the previous supercritical conditions in $\S 5.2$, there is no change in the shear-layer oscillation mode: the frequency is $S t_{D} \approx 0.31$ in both cases, corresponding to mode I (see figure 16). A low-frequency modulation is also measured with frequency $S t_{D}=0.008$ matching the prediction for mode ii (see table 3).

The formation of spanwise structures is again observed inside the cavity, with spanwise wavelength $\lambda / D=0.4$. Spectral analysis in the spanwise direction confirms this particular wavelength. In figure 17, it is clear that two different instabilities can be identified: first, a steady instability leading to the formation of a stationary cellular pattern near the upstream and bottom walls; and on top of it, an unsteady instability leading to the growth and decay of disturbances rotating inside the primary vortex. Here, because of the similarity in spanwise wavelength, we identified the steady mode with mode i from the linear analysis results. The unsteady mode, in addition to 
(a)

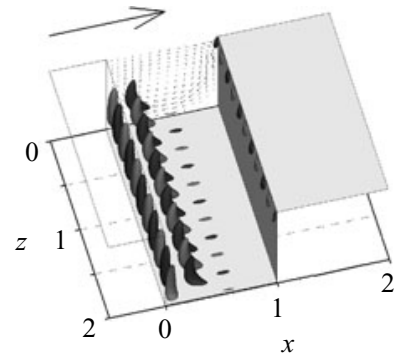

(d)

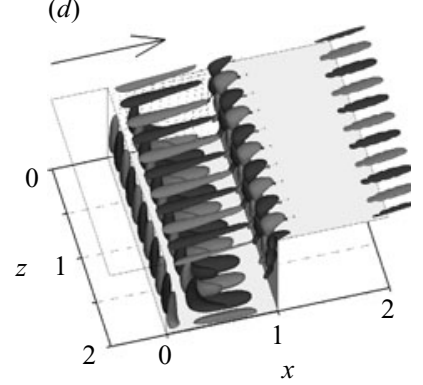

(b)

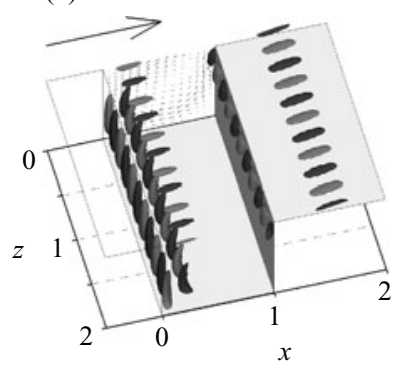

(e)

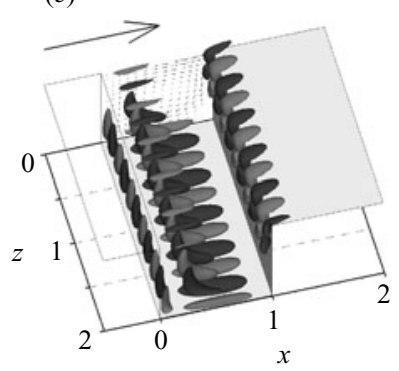

(c)
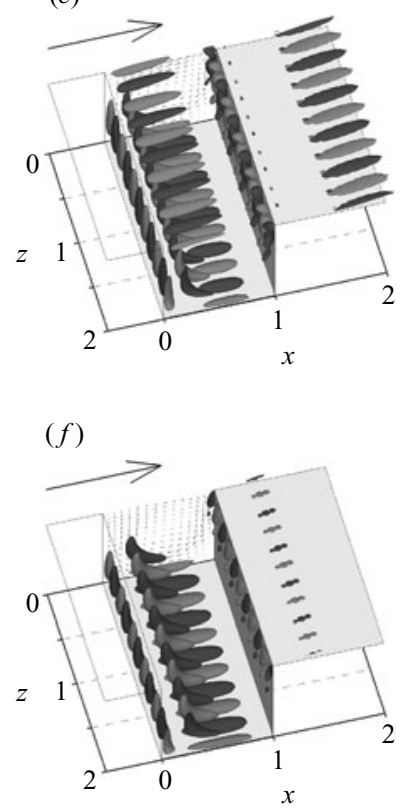

FIGURE 17. Visualisation of the three-dimensional flow for run H1Re300-3D at six different times $(a-f)$ corresponding to approximately one-sixth phase intervals of a period of the three-dimensional instability. The iso-surfaces represent the spanwise velocity levels $w / U=-0.005$ and $w / U=0.005$. The whole spanwise extent of the cavity is shown and the wavelength $\lambda / D=0.4$ of the instability is clear. The velocity vectors in the streamwise cross-section at $z=0$ are shown inside the cavity and once in the free stream for comparison.

its matching frequency, is also visually similar to mode ii from run 2M0325-3D in figure 11 , but its wavelength is 0.4 rather than the anticipated $\lambda / D=1$. As our linear analysis only captures the leading eigenvalue at a given spanwise wavelength, it is possible than mode ii does have a positive linear growth rate at $\lambda / D=0.4$ that is not measured because the growth rate of mode $i$ is larger. Under the present conditions, we suspect that both modes $\mathrm{i}$ and ii are selected. As a result of the presence of the steady mode, the time-averaged flow exhibits the same spanwise modulation (of wavelength $0.4 D$ ) in this case. The amplitude of the oscillations is also larger, as seen in figure 15.

\section{Connection with previous experimental and numerical results}

Measurements of the three-dimensional mode in cavity flow experiments face several challenges. The first difficulty arises from the influence of the sidewalls and end effects inherent in any experimental configuration. For cavities of small length-to-width ratio, the presence of the 'wall jet' and 'sidewall-induced three-dimensional vortices' (e.g. Armaly et al. 1983; Williams \& Baker 1997) can affect the flow up to several cavity depths in the spanwise direction away from the sidewalls and therefore suppress the three-dimensional instability (e.g. Albensoeder \& Kuhlmann 2006). Likewise, observations of three-dimensionality in the mean flow (e.g. Ahuja \& Mendoza 1995) are in general caused by spillage of flow over the sides into the cavity, and are therefore not related to the three-dimensional instability we identify. 
Asymmetry of the mean flow has also been reported in the experiment by Forestier et al. (2003) and reproduced with LES by Larchevêque, Sagaut \& Labbé (2007). In the LES, spanwise-non-uniform mean flows persisted even when periodic boundary conditions were used. Centrifugal instability was cited as a potential mechanism for the observed three-dimensionality. Unfortunately, for the same $L / D=2$, our analysis (at lower $R e$ ) would suggest that the centrifugal instability is oscillatory rather than stationary, and most amplified at a smaller wavelength than they reported $(\lambda \approx D$ as opposed to $\lambda=2.4 D$ ). Nevertheless, owing to the difference in Reynolds number it is not possible to make a definitive connection between their observations and the centrifugal instability we observe.

For wide cavities, three-dimensional structures have been observed by several researchers. In a water channel experiment, Rockwell \& Knisely (1980) identified a spanwise wavy structure emerging in the shear layer near the cavity trailing edge. Likewise, the high-Reynolds-number wind tunnel experiments by Maull \& East (1963) show, under certain conditions, regular steady 'cells' across the span of the internal cavity flow at low Mach numbers. While there is qualitative agreement with our findings, the results from these early experiments highlight some of the additional challenges related to the measurement of the three-dimensional instability: the threedimensional mode is in general weaker than the Rossiter mode and mainly active within the cavity, while most measurements focus on the shear layer; and the unsteady three-dimensional mode can be difficult to capture in the mean measurements because of its oscillatory nature. However, conclusive evidence of the three-dimensional instability we identify is found in recent experimental and numerical studies, discussed in the following sections.

\subsection{Interpretation of low-frequency modulation in experiments}

One feature of the unsteady three-dimensional mode that can be readily measured in experiment is its oscillation frequency. As previously discussed, this frequency is one order of magnitude lower than the typical Rossiter mode frequencies. As a results, it may have been unresolved or misinterpreted as being due to facility-dependent effects (e.g. fan noise) in some experiments.

Neary \& Stephanoff (1987) performed a set of water channel experiments for a cavity of aspect ratios $L / D=3.5$ and $W / D=21$, with a laminar incoming boundary layer $\left(L / \theta_{0} \approx 80\right)$. These conditions are comparable to our run series TK4M03: the visualization (in their figure 3 ) of the steady base flow for subcritical conditions at Reynolds number $R e_{D}=908$ shows a striking resemblance with our results in figure 7(c) obtained at $R e_{D}=980$ and $L / \theta_{0}=60$. Their results showed the presence of a transverse wave on the primary vortex of the cavity. As the Reynolds number is increased, stronger interactions between the shear layer and primary vortex are observed, with rapid growth and decay of the pressure oscillations. This feature indicates a Reynolds number dependence of the three-dimensional mode, similar to our linear results. Confirmation of the low frequency of the three-dimensional oscillation we obtain is also suggested by their observations. They identified a primary frequency $f_{1}$ that corresponds to the (Rossiter) shear-layer structures and a secondary frequency $f_{2}$ that they associate with the three-dimensional waviness. The frequency $f_{2}$ is close to $f_{1}$, but a re-examination of time-histories of pressure actually show a long-period modulation of the frequency $f_{1}$. Thus it would appear to us that the $f_{2}$ frequency is really just an interaction between a low frequency $f_{3 D}$ and the primary frequency $f_{1}$. From the reported frequency values for their so-called 'regime II', the frequency $f_{3 D} \approx f_{1}-f_{2}$ was estimated as $f_{3 D} D / U=0.023$. The value obtained 
here at $R e_{D}=2560$ is in excellent agreement with the frequency $S t_{D}=0.026$ of the three-dimensional instability identified in the linear analysis of run TK4M03Re65.

More recently, Cattafesta et al. (1998) and Kegerise et al. (2004) investigated the presence of multiple distinct peaks in the pressure spectrum of oscillating cavity flows with a turbulent incoming boundary layer. Their detailed frequency analysis of the unsteady pressure signals inside the cavity showed the presence of multiple Rossiter modes, in good agreement with expected frequencies. They also reported that these modes experienced low-frequency amplitude modulation. While they were able to interpret other peaks in the spectrum as quadratic nonlinear interactions between Rossiter modes, they concluded that these interactions were not the cause of the low-frequency component. Based on their conclusions and our results, we argue here that the low-frequency mode observed in their experiment corresponds to the threedimensional centrifugal instability identified in our work. The reported frequency for the cavity $L / D=2$ at $M=0.4$ and $R e_{L}=1.5 \times 10^{6}$ is $f_{3 D} D / U \approx 0.011$. Keeping in mind that an increase in Reynolds number was shown to cause a moderate decrease of the three-dimensional mode frequency, and that the boundary layer properties had little effect on the mode features, this result compares well with the range of frequencies $0.015 \leqslant S t_{D} \leqslant 0.026$ obtained for cavities with the same aspect ratio at $M=0.325$.

\subsection{Visual evidence of the three-dimensional mode}

The most compelling evidence of the three-dimensional centrifugal instability is found in the recent work of Faure et al. (2007). They performed low-speed experiments for open cavities of aspect ratio $L / D=0.5$ to 2 at medium Reynolds numbers, with laminar incoming boundary layers $(D / \theta \approx 35$, approximately constant according to a private communication with the authors). Smoke is used for flow visualizations. The cases with cavities of aspect ratio 1 and 2 are of particular interest since their experimental conditions (apart from the Mach number) match the range of parameters in our study.

For $L / D=1$, they report weak shear-layer oscillations and a single vortex filling the whole cavity. As the Reynolds number is increased, they observe the formation of 'mushroom-like counter-rotating cells' near the upstream wall and symmetrically at the downstream wall. Their visualization of the flow at a cross-section inside the cavity clearly shows a periodic spanwise pattern similar to our results in figure 18 . The inner spanwise flow caused by the sidewalls is also detected but the cavity spanto-depth ratio $W / D=6$ is large enough that the central section of the experiment exhibits periodic structures away from the sides. They report that these structures were not stationary and could not be identified for an external velocity below a certain critical value corresponding to $R e=4030$. This result matches the critical conditions $R e=4060$ we estimated for cavities with $L / D=1$ at low Mach number (see table 1). Additionally, for an external velocity $U_{e}=2.09 \mathrm{~m} \mathrm{~s}^{-1}(R e=6960$ matching exactly the Reynolds number used in our simulations), their measure of the distance between two pairs of structures gives a spanwise wavelength $\lambda=0.022 \mathrm{~m}$ or $\lambda / D=0.44$, again in excellent agreement with our conclusions from the linear analysis $(\lambda / D=0.4$ for run H1M03Re300) and the nonlinear three-dimensional simulation $(\lambda / D=0.4$ for run H1Re300-3D).

Similar results are reported for the $L / D=2$ cavity, but the structures are very unsteady in that case and the wavelength was not measured. The visualization of a cross-section of the flow suggests a larger wavelength that for the previous case, as fewer structures are present. These observations are consistent with our findings that 


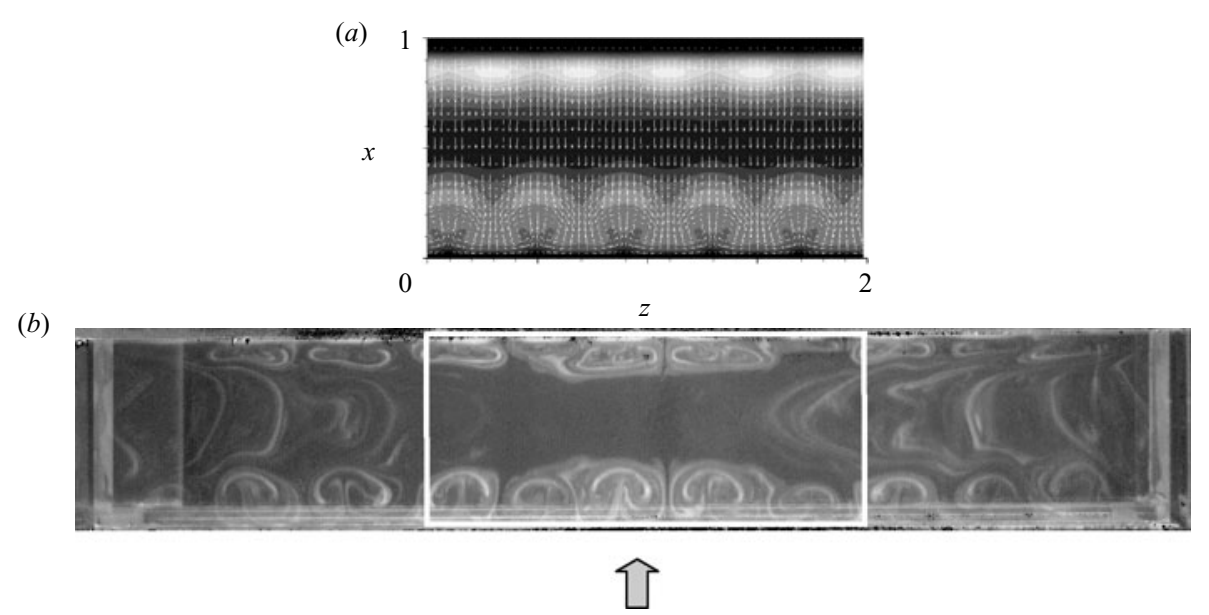

Flow direction

FIGURE 18. Comparison of the spanwise structures from the numerical simulations and experiments. (a) Equally spaced contours of the velocity magnitude between $\|\boldsymbol{u}\| / U=0.005$ and 0.06 in the cross-section $y / D=-0.6$, for run H1Re300-3D. The in-plane velocity vectors are also shown. (b) Figure 11(c) from Faure et al. (2007). The white box corresponds to the domain of the numerical simulations.

only an oscillatory three-dimensional mode is present for the longer cavity. Coupled with larger oscillations of the shear layer, the mode is more difficult to capture accurately. In contrast, the weaker shear-layer oscillation and the presence of both steady and oscillatory modes in smaller cavities lead to a clearer visualization of the instability.

Regarding the instability mechanism, Faure et al. (2007) related the threedimensional structures to the primary vortex inside the cavity and interpreted them in terms of Görtler vortices. The Görtler problem is a classical example of centrifugal instability (e.g. Drazin \& Reid 1981), and is typically studied for the onset of instability in boundary layers along a concave wall. While the two mechanisms are related, we argue here that the origin of three-dimensional modes is a generic centrifugal instability connected with the recirculating vortical flow inside the cavity, rather than instabilities in the boundary layers on the cavity walls.

\subsection{Connection with previous numerical simulations}

Podvin et al. (2006) and Chang et al. (2006) also reported the formation of spanwise structures and low-frequency modulations, for incompressible flows over open cavities. Similar to our work, both numerical studies focused on a cavity of aspect ratio $L / D=2$ with laminar incoming boundary layer and periodic conditions in the spanwise direction.

Chang et al. (2006) performed LES at $R e_{D}=3360$, with $L / \theta_{0}=69.5$. Under these conditions, they reported that the shear layer is oscillating with frequency corresponding to the second mode and that 'very low-frequency modulations are clearly observed' in time-history of the velocity. From the time series and power spectra (in their figures 6 and 7 respectively), we estimated the modulation frequency as $S t_{D}=f D / U \approx 0.025-0.03$. These values are similar to the frequency 0.026 in our compressible simulations with comparable conditions (i.e. run 2M06-3D). While they suspected these features are 'a consequence of the shear layer interaction with the trailing-edge and with the recirculating motions inside the cavity', we identify the centrifugal instability mechanism as the cause of the low-frequency modulation. 
Spanwise coherent structures, referred to as 'spanwise vortices', are also observed in their work, but values of the spanwise wavelength are not reported. Based on the visualization of these structures in their figure 8, approximately six pairs of these spanwise vortices can be accounted for along the cavity span, which is $W / D=6$ in their case. Therefore, the estimated spanwise wavelength of the instability $\lambda / D \approx 1$ matches our results.

Using proper orthogonal decomposition (POD), Podvin et al. (2006) investigated cavity flows at $R e_{D}=4000$, with $L / \theta_{0} \approx 75$. They related the first two most energetic POD modes to the shear-layer instabilities, and the next three modes (significantly less energetic) to 'fluctuating vortex motions inside the cavity'. The visualization of these latter modes in their figure 4 shows strong similarity with the threedimensional eigenmode in our figure 6 . They reported that all five modes exhibit a spanwise fluctuation of wavelength $\lambda / D \approx 1.4$, which is comparable to our results. They suggested that this particular wavelength corresponds to a secondary instability of the shear layer. Here, we again interpret the wavelength selection in terms of the centrifugal instability mechanism. Also, the higher-order modes have an identical low oscillation frequency, but the precise value is not reported, From the time spectra in their figure 8 , we estimated the frequency as $f D / U=0.021$, again in agreement with the three-dimensional mode frequency.

\section{Concluding remarks}

Two- and three-dimensional global instabilities of compressible flow over open cavities were studied using direct numerical simulations. We considered cavities that are homogeneous in the spanwise direction (corresponding to cavities that are wide compared to their depth or length) at subsonic Mach numbers.

Our linear stability analysis identified, for the first time, three-dimensional instabilities taking the form of disturbances growing in the recirculating vortical flow within the cavity. It should be noted that these instabilities would not be accessible to classical linear stability theory of parallel flows.

For cavities of aspect ratio $L / D=2$ and 4 , the three-dimensional instability has a spanwise wavelength scaling with $D$, the cavity depth, and a frequency of oscillation about one order of magnitude smaller than typical two-dimensional shear-layer (Rossiter) oscillations. We showed that the three-dimensional mode frequency is related to the time for disturbances to advect around the recirculating region.

A steady mode of smaller spanwise wavelength $0.4 D$ was also identified for a shorter cavity with $L / D=1$. We argued that the specific properties of the three-dimensional mode for the square cavity are related to the primary vortex that occupies the whole cavity in that particular configuration.

The mode properties are by and large unaffected by Mach number over a subsonic range up to 0.6. It is not expected that they would be significantly altered by compressibility even for cavities with much larger $M$, since the instability is active within the cavity where the flow speed is much lower than the free-stream value. In contrast, the influence of the Reynolds number is significant, as the growth rate of the three-dimensional mode increases with $R e$. Critical conditions for the onset of the three-dimensional instability were estimated.

Rayleigh's circulation criterion was computed for the two-dimensional steady base flow and showed that, in the inviscid limit, the flow will develop a three-dimensional centrifugal instability in the primary vortex inside the cavity. Therefore, we argued that the main mechanism behind these global three-dimensional instabilities is the generic centrifugal instability associated with the closed streamlines in the recirculating vortical flow near the downstream cavity wall. Such vortices are ubiquitous in both 
the low-Reynolds-number flows considered here and also (in a time-averaged sense) at much higher Reynolds numbers. The centrifugal instability is similar to the one previously identified in flows over a backward-facing step, lid-driven-cavity flows and Couette flows.

Direct numerical simulations of the nonlinear Navier-Stokes equations were performed and confirmed the three-dimensional features of the flow. Oscillating (and, for the square cavity, steady) cellular patterns formed in the recirculating zone inside the cavity. These observations were in agreement with the results of linear stability analysis, both in terms of spanwise structure and oscillation frequency. Comparison with recent experiments by Faure et al. (2007) at low Reynolds number also confirmed our findings.

For supercritical conditions, the shear-layer oscillations exhibit a low-frequency modulation due to the presence of the three-dimensional instability. Similar modulation and low-frequency components in the spectrum of oscillating cavity flows are reported in both incompressible and compressible experiments. Evidence of that low frequency was also found in previous numerical studies. We argue that these observations are related to the centrifugal instability that we identified.

As different distinctive properties of the three-dimensional centrifugal instability have been observed in both high-Reynolds-numbers flows and experiments with subsonic speed up to $M=0.6$, we conclude that three-dimensional modes are likely to exist in open cavity flows for practical applications.

This work was supported by AFOSR under grant F49620-02-1-0362. Computer time was provided in part by the Department of Defense High Performance Computing centres. The authors acknowledge contributions to the linear stability methodology from Professor Vassilis Theofilis, and would like to thank Professor Thierry Faure and his collaborators for allowing us to reproduce their experimental results.

\section{Appendix}

Table 4 lists the simulation parameters and stability characteristics for each run.

\begin{tabular}{|c|c|c|c|c|c|c|c|c|c|}
\hline \multirow[b]{2}{*}{ Run } & \multicolumn{5}{|c|}{ Parameter } & \multicolumn{4}{|c|}{ Stability } \\
\hline & $L / D$ & $M$ & $L / \theta_{0}$ & $R e_{\theta}$ & $R e_{D}$ & $2 \mathrm{D}$ & 3D linear & $\lambda / D$ & $S t_{D}$ \\
\hline H1M03Re150 & 1 & 0.3 & 23.2 & 150 & 3480 & NO & NO & & \\
\hline H1M03Re300 & 1 & 0.3 & 23.2 & 300 & 6960 & NO & $\mathrm{CI}$ & 0.4 & 0 \\
\hline H1 & 1 & 0.6 & 23.2 & 86.3 & 2000 & NO & NO & & \\
\hline $\mathrm{H} 1 \mathrm{Re} 110$ & 1 & 0.6 & 23.2 & 110 & 2550 & $\mathrm{NO}$ & NO & & \\
\hline H1Re140 & 1 & 0.6 & 23.2 & 140 & 3250 & NO & NO & & \\
\hline H1Re200 & 1 & 0.6 & 23.2 & 200 & 4640 & $\mathrm{NO}$ & CI & 0.5 & 0 \\
\hline H1Re300 & 1 & 0.6 & 23.2 & 300 & 6960 & SL & & & \\
\hline 2M01 & 2 & 0.1 & 52.8 & 56.8 & 1500 & $\mathrm{NO}$ & CI & 1 & 0.025 \\
\hline 2M01Re80 & 2 & 0.1 & 52.8 & 80 & 2110 & NO & CI & 1 & 0.023 \\
\hline 2M03Re35 & 2 & 0.3 & 52.8 & 35 & 925 & $\mathrm{NO}$ & NO & & \\
\hline $2 \mathrm{MO} 3$ & 2 & 0.3 & 52.8 & 56.8 & 1500 & $\mathrm{NO}$ & CI & 1 & 0.025 \\
\hline 2M03Re65 & 2 & 0.3 & 52.8 & 65 & 1715 & $\mathrm{NO}$ & CI & 1 & 0.025 \\
\hline 2M03Re80 & 2 & 0.3 & 52.8 & 80 & 2110 & SL & & & \\
\hline 2M0325 & 2 & 0.325 & 52.8 & 56.8 & 1500 & $\mathrm{NO}$ & CI & 1 & 0.025 \\
\hline 2M035 & 2 & 0.35 & 52.8 & 56.8 & 1500 & $\mathrm{NO}$ & CI & 1 & 0.026 \\
\hline 2M035Re60 & 2 & 0.35 & 52.8 & 60 & 1585 & SL & & & \\
\hline 2M0365 & 2 & 0.365 & 52.8 & 56.8 & 1500 & SL & & & \\
\hline
\end{tabular}

TABLE 4. Continued on facing page 


\begin{tabular}{|c|c|c|c|c|c|c|c|c|c|}
\hline \multirow[b]{2}{*}{ Run } & \multicolumn{5}{|c|}{ Parameter } & \multicolumn{4}{|c|}{ Stability } \\
\hline & $L / D$ & $M$ & $L / \theta_{0}$ & $R e_{\theta}$ & $\operatorname{Re}_{D}$ & $2 \mathrm{D}$ & 3D linear & $\lambda / D$ & $S t_{D}$ \\
\hline 2M038Re50 & 2 & 0.38 & 52.8 & 50 & 1320 & $\mathrm{NO}$ & $\mathrm{CI}$ & 1 & 0.025 \\
\hline 2M04Re50 & 2 & 0.4 & 52.8 & 50 & 1320 & SL & & & \\
\hline 2M045Re50 & 2 & 0.45 & 52.8 & 50 & 1320 & SL & & & \\
\hline 2M05Re35 & 2 & 0.5 & 52.8 & 35 & 925 & SL & & & \\
\hline $2 \mathrm{M} 06$ & 2 & 0.6 & 52.8 & 56.8 & 1500 & SL & & & \\
\hline TK2M0325 & 2 & 0.325 & 30.1 & 56.8 & 855 & $\mathrm{NO}$ & NO & & \\
\hline TK2M0325Re80 & 2 & 0.325 & 30.1 & 80 & 1205 & NO & NO & & \\
\hline TK2M0325Re100 & 2 & 0.325 & 30.1 & 100 & 1505 & NO & NO & & \\
\hline TK2M0325Re140 & 2 & 0.325 & 30.1 & 140 & 2110 & $\mathrm{NO}$ & CI & 1 & 0.015 \\
\hline TK2M06 & 2 & 0.6 & 30.1 & 56.8 & 855 & NO & NO & & \\
\hline TK2M06Re80 & 2 & 0.6 & 30.1 & 80 & 1205 & $\mathrm{NO}$ & NO & & \\
\hline TK2M06Re140 & 2 & 0.6 & 30.1 & 140 & 2110 & $\mathrm{NO}$ & $\mathrm{CI}$ & 1 & 0.016 \\
\hline TK4M03 & 4 & 0.3 & 60.2 & 50 & 755 & $\mathrm{NO}$ & NO & & \\
\hline TK4M03Re65 & 4 & 0.3 & 60.2 & 65 & 980 & NO & CI & 1.25 & 0.026 \\
\hline TK4M03Re80 & 4 & 0.3 & 60.2 & 80 & 1205 & SL & & & \\
\hline TK4M06Re30 & 4 & 0.6 & 60.2 & 30 & 450 & $\mathrm{NO}$ & NO & & \\
\hline TK4M06Re40 & 4 & 0.6 & 60.2 & 40 & 600 & SL & & & \\
\hline TK4M06 & 4 & 0.6 & 60.2 & 50 & 755 & SL & & & \\
\hline 4M03Re200 & 4 & 0.3 & 30.1 & 200 & 1505 & NO & NO & & \\
\hline 4M03Re400 & 4 & 0.3 & 30.1 & 400 & 3010 & $\mathrm{NO}$ & $\mathrm{CI}$ & 1.25 & 0.011 \\
\hline
\end{tabular}

TABLE 4. Parameters and stability of the different two-dimensional (2D) and three-dimensional (3D) linear simulations. Abbreviations for stability are: $\mathrm{NO}=$ no oscillations, $\mathrm{SL}=$ shear-layer mode, $\mathrm{CI}=$ three-dimensional centrifugal instability. For supercritical conditions, the linear stability results are not available. For the centrifugal instability, the spanwise wavelength $\lambda / D$ and the oscillation frequency $S t_{D}=\omega D / 2 \pi U$ of the dominant mode are reported.

\section{REFERENCES}

Ahuja, K. K. \& Mendoza, J. 1995 Effects of cavity dimensions, boundary layer and temperature on cavity noise with emphasis on benchmark data to validate computational aeroacoustic codes. Tech. Rep. CR-4653. NASA.

Aidun, C. K., Triantafillopoulos, N. G. \& Benson, J. D. 1991 Global stability of a lid-driven cavity with throughflow: Flow visualization studies. Phys. Fluids A 3, 2081-2091.

Albensoeder, S. \& Kuhlmann, H. C. 2006 Nonlinear three-dimensional flow in the lid-driven square cavity. J. Fluid Mech. 569, 465-480.

Albensoeder, S., Kuhlmann, H. C. \& Rath, H. J. 2001 Three-dimensional centrifugal-flow instabilities in the lid-driven-cavity problem. Phys. Fluids 13, 121-135.

Alvarez, J., Kerschen, E. \& Tumin, A. 2004 A theoretical model for cavity acoustic resonances in subsonic flow. AIAA Paper 2004-2845.

Armaly, B. F, Durst, F., Pereira, J. C. F. \& Schönung, B. 1983 Experimental and theoritical investigation of backward-facing step flow. J. Fluid Mech. 127, 473-496.

BARKLEY, D., Gomes, G. M \& Henderson, R. D. 2002 Three-dimensional instability in flow over a backward-facing step. J. Fluid Mech. 473, 167-190.

BAYLY, B. J. 1988 Three-dimensional centrifugal-type instabilities in inviscid two-dimensional flows. Phys. Fluids 31, 56-64.

Benson, J. D. \& Aidun, C. K. 1992 Transition to unsteady nonperiodic state in a through-flow lid-driven cavity. Phys. Fluids A 4, 2316-2319.

BRÈs, G. A. 2007 Numerical simulations of three-dimensional instabilities in cavity flows. PhD thesis, California Institute of Technology.

BRÈs, G. A. \& Colonius, T. 2007 Three-dimensional linear stability analysis of cavity flows. AIAA Paper 2007-1126. 
Cattafesta III, L. N., Garg, S., Kegerise, M. S. \& Jones, G. S. 1998 Experiments on compressible flow-induced cavity oscillations. AIAA Paper 98-2912.

Cattafesta III, L. N., Shukla, D., Garg, S. \& Ross, J. A. 1999 Development of an adaptive weapons-bay suppression system. AIAA Paper 99-1901.

Chang, K., Constantinescu, G. \& Park, S. 2006 Analysis of the flow and mass transfer processes for the incompressible flow past an open cavity with a laminar and a fully turbulent incoming boundary layer. J. Fluid Mech. 561, 113-145.

Chatellier, L., J., LAumONier \& Gervais, Y. 2006 Theoretical and experimental investigation of low Mach number turbulent cavity flows. Exps. Fluids 36, 728-740.

Colonius, T. 2001 An overview of simulation, modeling, and active control of flow/acoustic resonance in open cavities. AIAA Paper 2001-0076.

Colonius, T., Lele, S. K. \& Moin, P. 1993 Boundary conditions for direct computation of aerodynamic sound. AIAA J. 31, 1547-1582.

Ding, Y. \& Kawahara, M. 1999 Three-dimensional linear stability of incompressible viscous flow using the finite element method. Intl J. Numer. Meth. Fluids 31, 451-479.

DiPrima, R. C., Eagles, P. M. \& NG, B. S. 1984 The effect of radius ratio on the stability of Couette flow and Taylor vortex flow. Phys. Fluids 27, 2403-2411.

Drazin, P. G. \& Reid, W. H. 1981 Hydrodynamic Stability. Cambridge University Press.

Faure, T. M., Adrianos, P., Lusseyran, F. \& Pastur, L. 2007 Visualizations of the flow inside an open cavity at medium range Reynolds numbers. Exps. Fluids 42, 169-184.

Forestier, N., JACQuin, L. \& GefFroy, P. 2003 The mixing layer over a deep cavity at highsubsonic speed. J. Fluid Mech. 475, 101-145.

Freund, J. B. 1997 Proposed inflow/outflow boundary condition for direct computation of aerodynamic sound. AIAA J. 35, 740-742.

Frigo, M. \& Johnson, S. G. 2007 FFTW library. Website: http://www.fftw.org.

Gharib, M. \& Roshko, A. 1987 The effect of flow oscillations on cavity drag. J. Fluid Mech. 177, $501-530$.

Kegerise, M. A., Spina, E. F., Garg, S. \& Cattafesta, III, L. N. 2004 Mode-switching and nonlinear effects in compressible flow over a cavity. Phys. Fluids 16, 678-687.

LarchevêQue, L., Sagaut, P. \& LabbÉ, O. 2007 Large-eddy simulation of a subsonic cavity flow including asymmetric three-dimensional effects. J. Fluid Mech. 577, 105-126.

Larchevêque, L., Sagaut, P., LÊ, T. \& Comte, P. 2004 Large-eddy simulation of a compressible flow in a three-dimensional open cavity at high Reynolds number. J. Fluid Mech. 516, 265-301.

Larchevêque, L., Sagaut, P., Mary, I., Labbé, O. \& Comte, P. 2003 Large-eddy simulation of a compressible flow past a deep cavity. Phys. Fluids 15, 193-210.

Lehoucq, R., Maschhoff, K., Sorensen, D. \& Yang, C. 2007 ARPACK software. Website: http://www.caam.rice.edu/software/ARPACK/.

Lele, S. K. 1992 Compact finite difference scheme with spectral-like resolution. J. Comput. Phys. $103,16-42$.

Maull, D. J. \& East, L. F. 1963 Three-dimensional flow in cavities. J. Fluid Mech. 16, 620-632.

NeARY, M. D. \& StephanofF, M. D. 1987 Shear-layer-driven transition in a rectangular cavity. Phys. Fluids 30, 2936-2946.

Podvin, B., Fraigneau, Y., Lusseyran, F. \& Gougat, P. 2006 A reconstruction method for the flow past an open cavity. Trans. ASME: J. Fluids Engng 128, 531-540.

Poinsot, T. J. \& Lele, S. K. 1992 Boundary conditions for direct simulations of compressible viscous flows. J. Comput. Phys. 101, 104-129.

Ramanan, N. \& Homsy, G. M. 1994 Linear stability of lid-driven cavity flow. Phys. Fluids 6, 2690-2701.

Rizzetta, D. P. \& Visbal, M. R. 2003 Large-eddy simulation od supersonic cavity flowfields including flow control. AIAA J. 41, 1452-1462.

Rockwell, D. \& KNisely, C. 1980 Observations of the three-dimensional nature of unstable flow past a cavity. Phys. Fluids 23, 425-431.

Rockwell, D. \& NAUdASCHER, E. 1978 Review - self-sustaining oscillations of flow past cavities. Trans. ASME: J. Fluids Engng 100.

Rossiter, J. E. 1964 Wind-tunnel experiments on the flow over rectangular cavities at subsonic and transonic speeds. Tech. Rep. 3438. ARC. 
Rowley, C. W., Colonius, T. \& Basu, A. J. 2002 On self-sustained oscillations in two-dimensional compressible flow over rectangular cavities. J. Fluid Mech. 455, 315-346.

Rowley, C. W. \& Williams, D. R. 2006 Dynamics and control of high-Reynolds-number flow over open cavities. Annu. Rev. Fluid Mech. 38, 251-276.

Rowley, C. W., Williams, D. R., Colonius, T., Murray, R. M. \& MacMynowski, D. G. 2006 Linear models for control of cavity flow oscillations. J. Fluid Mech. 547, 317-330.

SAROHIA, V. 1975 Experimental and analytical investigation of oscillations in flows over cavities. $\mathrm{PhD}$ thesis, California Institute of Technology.

Theofilis, V. \& Colonius, T. 2003 An algorithm for the recovery of 2- and 3-D biglobal instabilities of compressible flow over 2-D open cavities. AIAA Paper 2003-4143.

Theofilis, V., Duck, P. W. \& Owen, J. 2004 Viscous linear stability analysis of rectangular duct and cavity flows. J. Fluid Mech. 505, 249-286.

Thompson, K. W. 1990 Time-dependent boundary conditions for hyperbolic systems, II. J. Comput. Phys. 89, 439-461.

Williams, P. T. \& BAKER, A. J. 1997 Numerical simulations of laminar flow over a 3D backwardfacing step. Intl J. Numer. Meth. Fluids 24, 1159-1183. 